\title{
Directividad de la fuente sísmica en el terremoto de Lorca del 11 de mayo de 2011
}

\author{
JUAN RUEDA NÚÑEZ ${ }^{1,2}$, JULIO MEZCUA RODRÍGUEZ ${ }^{1}$ \\ \& ROSA MARIA GARCÍA BLANCO ${ }^{2}$ \\ ${ }^{1}$ Instituto Geográfico Nacional \\ ${ }^{2}$ Universidad Politécnica de Madrid \\ jjrueda@fomento.es
}

Recibido: 20/06/2012

Aceptado: 14/09/2012

\section{Resumen}

El estudio de la fuente sísmica del terremoto de Lorca del 11 de mayo de 2011, 5,1 Mw, permite concluir que un efecto de directividad de la fuente sísmica es el principal causante de las altas aceleraciones registradas en la ciudad de Lorca responsables de los cuantiosos daños, junto con posibles efectos de amplificación por el tipo de suelo.

Se ha recalculado el tensor momento sísmico de los tres terremotos principales de la serie, asociándose los mismos a la falla de Alhama de Murcia. Se ha calculado la distribución del deslizamiento en el plano de falla, obteniéndose una ruptura unilateral de velocidad $2,4 \mathrm{~km} / \mathrm{s}$ dirigida desde el hipocentro a la ciudad de Lorca, con un deslizamiento máximo de $17,5 \mathrm{~cm}$ a $2 \mathrm{~km}$ de profundidad. El análisis de la función fuente realizado con el método de las funciones empíricas de Green y la relación de amplitudes Wood-Anderson entre el terremoto principal y réplicas de baja magnitud, muestra también un claro efecto de directividad en la dirección de la falla. Se ha extraído un pulso de directividad en el registro del acelerógrafo de Lorca de $33,2 \mathrm{~cm} / \mathrm{s}$, con una frecuencia de 1,5 Hz, que es considerado el principal responsable de los daños.

Palabras clave: Terremoto de Lorca, fuente sísmica, tensor momento sísmico, deslizamiento, directividad de la ruptura, pulso de directividad.

\footnotetext{
Seismic source directivity on May 11, 2011 Lorca earthquake

\section{Abstract}

The seismic source study performed to the Lorca 11 May 2011, 5.1 Mw earthquake allow us to conclude that a directivity effect is responsible of much of the high acceleration recorded at the city of Lorca which causes great damage.

A new moment seismic tensor inversion has been obtained for the three main events of the earthquake series associated to the Alhama de Murcia Fault. A slip distribution over the fault plane has been obtained, showing a unilateral rupture in hypocenter-Lorca direction with a rupture velocity of $2.4 \mathrm{~km} / \mathrm{s}$ with a maximum displacement of $17.5 \mathrm{~cm}$ at $2 \mathrm{~km}$ depth. The source time function analysis performed though Empirical Green Functions and the Wood-Anderson Amplitudes relation between main event and lower magnitude aftershocks shows also a clear directivity effect in the fault direction. A directivity pulse has been extracted from the strong ground motion instrument at Lorca of $33.2 \mathrm{~cm} / \mathrm{s}$ centered at a $1.5 \mathrm{~Hz}$ which we considered main responsible of the earthquake damage.
}

Keywords: Lorca earthquake, source function, seismic moment tensor, slip, rupture directivity, directivity pulse.

Sumario: Introducción. 1. Directividad de la fuente sísmica. 2. Obtención de las características la fuente sísmica mediante inversión de registros de banda ancha. 2.1. Tensor Momento Sísmico. 2.2. Inversión en una falla finita. Distribución del deslizamiento. 3. Análisis de la directividad. 
3.1. Extracción del pulso de directividad. 3.2. Función temporal en la fuente. 3.3. Relación de amplitudes. 4. Conclusiones. Agradecimientos. Referencias bibliográficas.

\section{Referencia normalizada}

Rueda, J., Mezcua, J. y García-Blanco, R.M. (2012). Directividad de la fuente sísmica en el terremoto de Lorca del 11 de mayo de 2011. Física de la tierra, Vol.24, 83-111.

\section{Introducción}

El 11 de mayo de 2011, a las 16:47 (T.U.), un terremoto de magnitud 5,1 Mw (lat $=37,7267 \mathrm{~N}$, lon $=1,6862 \mathrm{~W}, \mathrm{~h}=5 \mathrm{~km}$; IGN, 2011) sacudió de forma violenta la ciudad de Lorca. Según describieron los habitantes de la ciudad fue una sacudida de pequeña duración, pero con una gran intensidad. Algunas personas lo definieron como un "latigazo", otros como un "pulso". El terremoto fue precedido 1 hora 42 minutos antes por otro de magnitud 4,5 Mw (lat $=37,7216 \mathrm{~N}$, lon $=1,6990 \mathrm{~W}, \mathrm{~h}=5 \mathrm{~km}$; IGN, 2011), que también fue sentido causando alarma en la población y seguido por una serie de réplicas que se prolongó durante unos 2 meses.

El terremoto de magnitud 5,1 Mw, con epicentro localizado por el IGN a $5 \mathrm{~km}$ al NE de Lorca (Figura 1), causó 9 muertos, cientos de heridos y numerosísimos daños en la ciudad, con el colapso de alguna estructura de hormigón y daños irreparables en el patrimonio histórico. Algunas de las personas que habitaban los edificios que colapsaron salvaron la vida por haber desalojado los mismos por la alarma que causó el sismo premonitorio

Desde el punto de vista tectónico, los terremotos han ocurrido en el sector oriental de la cordillera Bética, en la región de contacto entre las zonas internas y externas que está formada por diferentes sistemas de fallas activas, intercaladas por cuencas sedimentarias del terciario y cuaternario y por pequeñas sierras. Una de estas fallas, en el interior de la zona interna, es la falla de Alhama de Murcia (Figura 1), de dirección NE-SO, comienza en las proximidades de Alcantarilla (Murcia) llegando hasta Góñar (Almería), Bousquet (1979). En el sector próximo a la ciudad de Lorca, la falla tiene movimiento de desgarre, con una componente inversa de buzamiento $50^{\circ}$ hacia el NO y una estructura compleja, tipo dúplex, que se complica con la interacción de la falla de Las Viñas, Martínez-Díaz (2002).

En este sector oriental de la cordillera Bética, en los últimos años han ocurrido varios terremotos de magnitud intermedia, pero con un gran nivel de daños: el 2 de febrero de 1999 el terremoto de Mula de magnitud 4,8 Mw, Buforn y Sanz de Galdeano (2001), Mancilla et al. (2002), Stich et al. (2003), Buforn et al. (2005); el 6 de agosto de 2002 en Bullas de magnitud 5,1 Mw, Stich et al. (2006), Buforn et al. (2005) y el 29 de enero de 2005 en La Paca con una magnitud de 4,7 Mw, Buforn et al. (2006), Benito et al. (2007). Los mecanismos focales de los terremotos de Bullas de 2002 y La Paca de 2005, se corresponden con fallas de desgarre. Para el terremoto de Mula de 1999, Mancilla et al. (2002) y Stich et al. (2003) obtienen mediante inversión del tensor momento sísmico un mecanismo en falla de desgarre asociado a la falla de Crevillente, mientras que Buforn y Sanz de 
Galdeano (2001) y Buforn et al. (2005) calculan con polaridades de la onda P un mecanismo en falla inversa asociado al accidente Cádiz-Alicante. En la zona más próxima a Lorca, solamente han ocurrido dos terremotos de los que se dispone de mecanismo focal, el 6 de junio de 1977 de magnitud 4,5 mbLg, Mezcua et al. (1984) y el 23 de agosto de 2000 con magnitud 3,8 mbLg, Martínez-Díaz et al. (2002); ambos con un mecanismo en falla normal son ejemplos de terremoto extensional en un régimen compresivo, como consecuencia de una posible extensión de la parte superficial de un bloque ante el empuje más profundo.

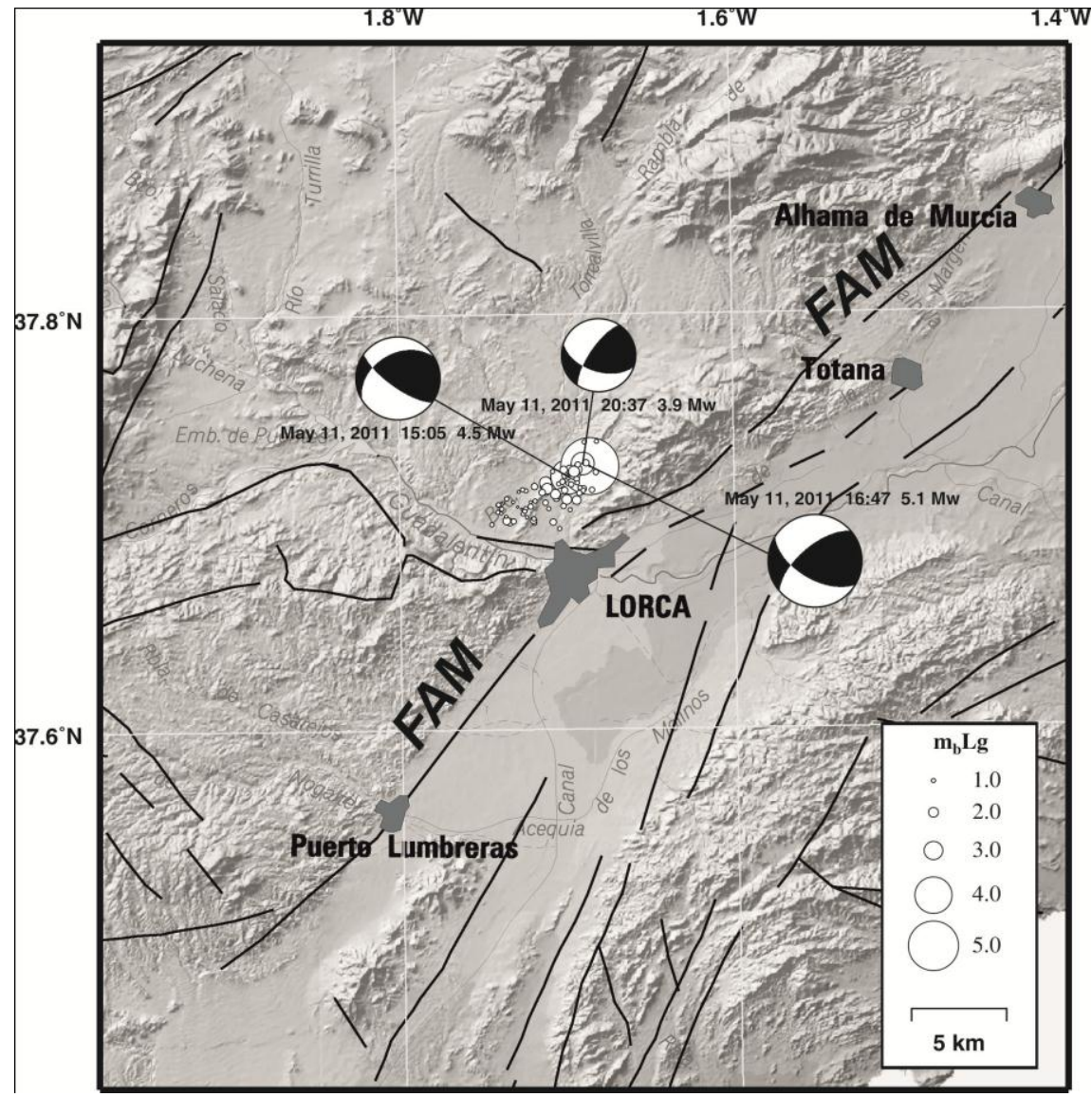

Fig. 1. Distribución epicentral del terremoto del 11 de mayo de 2011, su premonitorio y la serie de réplicas, IGN (2011), junto con los mecanismos focales calculados en este trabajo. Se incluyen las principales fracturas cartografiadas en la zona. FAM: falla de Alhama de Murcia. 
El terremoto de Lorca de 11 de mayo de 2011, localizado por el IGN $5 \mathrm{~km}$ al NE de la ciudad (Figura 1) (lat $=37,7267 \mathrm{~N}$, lon $=1,6882 \mathrm{~W}, \mathrm{~h}=5 \mathrm{~km}$; IGN, 2011), ha sido asociado a la falla de Alhama de Murcia desde el primer momento por IGME (2011) e IGN (2011), y en varios estudios posteriores por Frontera et al. (2011), Rueda et al. (2011), Vissers y Meijninger (2011), López-Comino et al. (2012), Martinez-Díaz et al. (2012) y Santoyo (2012), en base a distintos aspectos estudiados de la serie sísmica. En la Figura 1 podemos ver la distribución epicentral del terremoto principal, su premonitorio y la serie de réplicas una vez ha sido relocalizada, IGN (2011), junto con los mecanismos focales calculados en este trabajo.

Rueda et al. (2011) y López-Comino et al. (2012), establecen que una combinación del efecto de directividad de la ruptura, junto con el factor de amplificación del suelo son los posibles responsables de la alta aceleración registrada en Lorca, Figura 2, y por tanto de la gran cantidad de daños ocasionados por el terremoto. En este trabajo, realizamos un pormenorizado análisis sismológico de las características de la fuente sísmica, para intentar explicar, mediante un proceso de directividad, las grandes aceleraciones registradas en la ciudad de Lorca como consecuencia del terremoto del 11 de mayo de 2011.
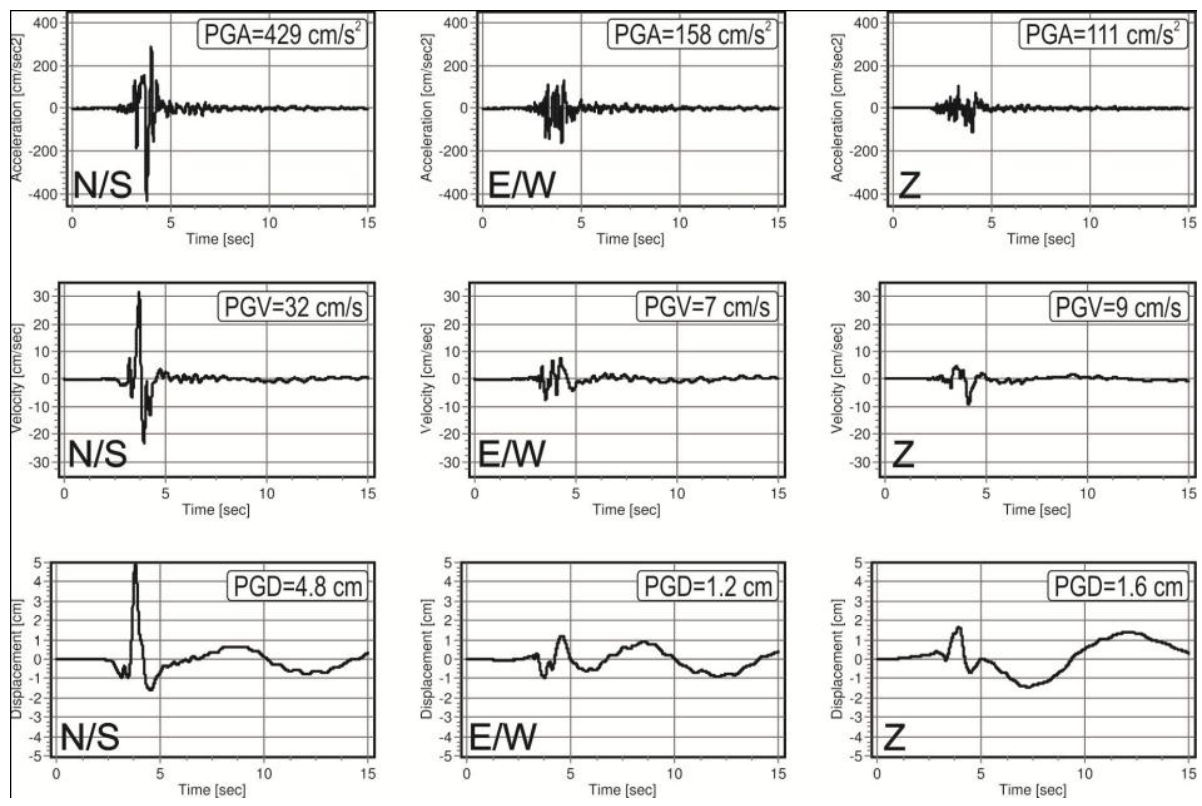

Fig. 2. Registros de aceleración, velocidad y desplazamiento en el acelerógrafo situado en la ciudad de Lorca, para el terremoto del 11 de mayo de 2011, 16:47 T.U. 5,1 Mw. Las componentes horizontales han sido rotadas $30^{\circ}$ al este para obtener las componentes NS y EO, ya que el instrumento que registró el terremoto no estaba orientado, IGN 2011. 


\section{Directividad de la fuente sísmica}

Para explicar la asimetría del patrón de radiación observada en grandes terremotos, Benioff (1955) utilizó un modelo de una fuente que se mueve con velocidad a lo largo de un segmento de falla (Figura 3). El término "directividad" fue asociado por primera vez a este tipo de ruptura en una falla por Ben-Menahem (1961) y se observa en la práctica cuando el patrón de radiación deja de ser simétrico. Esto ocurre con las fuentes puntuales, donde sus lóbulos pasan a ser más o menos extensos dependiendo de la dirección de ruptura a lo largo de la falla. Además, al moverse la fuente con una velocidad de ruptura determinada, la radiación se focaliza en la dirección del movimiento, en un efecto análogo al efecto Doppler que se presenta en las ondas acústicas.

Ben-Menahem y Singh (1972) desarrollaron patrones de radiación válidos para fuentes que se propagan en movimiento y que han sido posteriormente usados por numerosos autores para explicar los fenómenos de directividad observados. La amplitud y frecuencia de las ondas aumenta en la dirección de la propagación de la ruptura (resultante en A de la Figura 3) y disminuye en la dirección opuesta (resultante en B de la Figura 3).

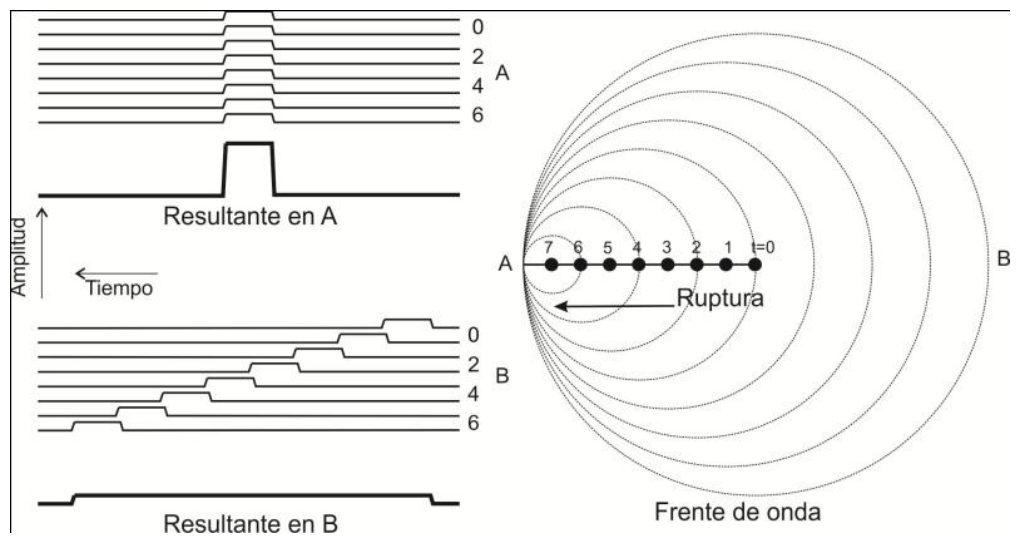

Fig. 3. Frente de onda correspondiente a una ruptura que avanza en una dirección. Resultante en un punto en la dirección del avance (A) con aumento de amplitud y frecuencia y en la dirección opuesta (B). Adaptado de Benioff (1955).

El fenómeno de la directividad ha sido observado incluso en terremotos de pequeña y moderada magnitud, con una velocidad de ruptura próxima a la velocidad de propagación de la onda S, Boatwright (2007), Seekins y Boatwright (2010) y Luo et al. (2010). Este fenómeno debe tenerse en cuenta en el cálculo de la peligrosidad sísmica, mediante la modificación de las relaciones empíricas de la atenuación, incluyendo en las mismas los efectos de la directividad de la ruptura, Somerville et al. (1997), o con la inclusión del pulso de directividad en el cálculo 
probabilístico de la peligrosidad sísmica, Tothong et al. (2007), Shahi y Baker (2010).

En el caso del terremoto de Lorca del 11 de mayo de 2011, existe una clara asimetría de los valores de aceleración registrados en las estaciones de la Red de Acelerógrafos del IGN (IGN, 2011). La distribución de daños también refleja esta característica: a distancias iguales al epicentro, los daños han sido mucho mayores en la dirección SO (posible dirección de avance de la ruptura y resultante en A de la Figura 3) que en cualquier otra, y particularmente mucho mayor que en la dirección contraria NE (resultante en B la Figura 3), donde los daños han sido escasos. El registro del acelerógrafo de Lorca, Figura 2, refleja una aceleración máxima en la componente $\mathrm{N} / \mathrm{S}$ de $429 \mathrm{~cm} / \mathrm{s}^{2}$, muy superior a lo que podrían predecir los modelos empíricos para una magnitud de 5,1 Mw. Esta aceleración es muy elevada, incluso si se añade el factor de amplificación para un emplazamiento con una Vs30 de $500 \mathrm{~m} / \mathrm{s}$, Navarro et al. (2008), que se corresponde con un suelo tipo C de la clasificación NEHRP, Martin (1994), o tipo II (roca muy fracturada, suelo granular denso o cohesivo duro) de la clasificación de la norma sismorresistente española, NCSE (2002).

Nos encontramos entonces ante un caso observado de una aceleración que a una distancia de $5 \mathrm{~km}$ del epicentro no puede explicarse solamente con un factor de amplificación del suelo, y por tanto es necesario investigar las características de la fuente, para comprobar si las causas de esta alta aceleración pudieran encontrarse en los efectos de una directividad de la ruptura desde el hipocentro hacia la ciudad de Lorca.

\section{Obtención de las características de la fuente sísmica mediante inversión de registros de banda ancha}

\subsection{Tensor Momento Sísmico}

A los pocos minutos de ocurrir un terremoto de importancia, el proceso automático implementado en el Instituto Geográfico Nacional por Rueda y Mezcua (2005) realiza una determinación rápida del TMS utilizando las tres primeras estaciones de banda ancha que han registrado el evento. Posteriormente, estas soluciones han sido recalculadas en este trabajo, utilizando todas las estaciones de banda ancha disponibles.

La descomposición del TMS nos permite obtener el momento sísmico escalar $M_{0}$ y por lo tanto la magnitud momento $\mathrm{Mw}$ y el mecanismo focal en el que se definen dos planos nodales candidatos a ser uno solo el plano de falla.

El método utilizado está basado en la inversión del sismograma completo en desplazamiento de baja frecuencia a distancia regional, suponiendo una fuente puntual con una función delta como función fuente, Dreger et al. (1998). Esta suposición puede considerarse válida para magnitudes menores de 7 en el rango de periodos de 10-20 s, Fukuyama y Dreger (2000). Las funciones de Green han sido determinadas mediante un método desarrollado por Saikia (1994) de frecuencia-número de onda, que usa la integración por el método de Filón y una 
aproximación polinómica de las funciones de Bessel. El modelo de tierra utilizado está basado en Mezcua y Martínez Solares (1983), al que de acuerdo con datos locales se le ha añadido una capa superficial de $2 \mathrm{~km}$ de espesor con una velocidad de 4,5 km/s. El proceso del cálculo del TMS mediante inversión a distancia regional, requiere el uso de modelos de tierra muy bien calibrados, Dreger y Helmberger (1991), Fan y Wallace (1991) y Walter (1993). Además, el método de inversión usando el sismograma completo es más sensible al modelo de tierra que a la localización. El análisis de sensibilidad realizado por Dreger y Helmberger (1993) muestra que los efectos de la no consideración de heterogeneidades laterales en el modelo no aparecen en las ondas de largo periodo, pero que estas sí son sensibles a la profundidad. La razón se encuentra en que las variaciones laterales se concentran en las capas más superficiales del modelo, donde las ondas internas son insensibles para periodos mayores de $10 \mathrm{~s}$.

Hemos calculado el TMS de los tres terremotos de mayor magnitud de la serie, esto es: el terremoto principal, el premonitorio y la mayor de sus réplicas. En la Figura 4 se representa la situación de todas las estaciones de banda ancha utilizadas en el cálculo del TMS, junto con el epicentro del terremoto principal.

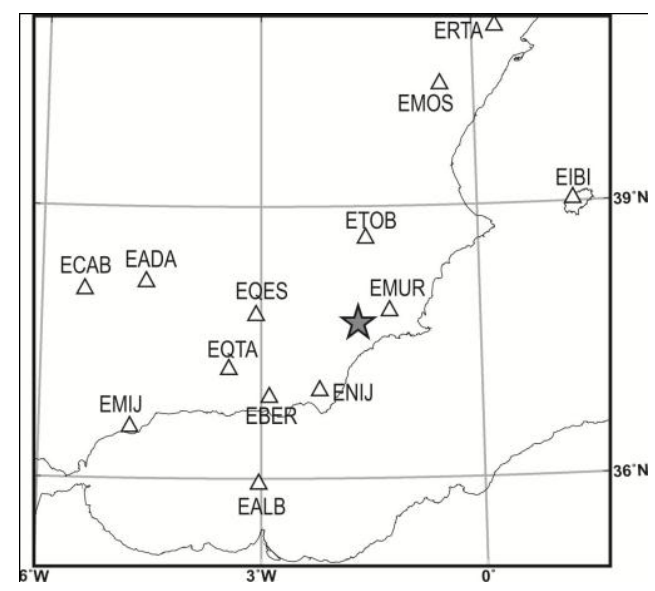

Fig. 4. Estaciones de banda ancha utilizadas en el cálculo del tensor momento sísmico y localización del terremoto del 11 de mayo de 2011 (estrella).

Antes de la inversión, sismogramas y funciones de Green han sido convertidos en desplazamiento y filtrados entre 0.02 y $0.05 \mathrm{~Hz}$ para conservar la señal de largo periodo. Con la inversión se ha obtenido el TMS completo, del que mediante descomposición se ha extraído la parte desviatoria. En la Tabla 1 figuran los parámetros de acimut, buzamiento y deslizamiento de los planos nodales, el momento sísmico escalar, magnitud momento, porcentajes de fuente de doble par y dipolo compensado y el parámetro de reducción de la varianza RV para cada 
uno de los tres TMS calculados. Este parámetro $R V$ define la calidad del ajuste entre los sismogramas reales y los sintéticos obtenidos a partir del TMS calculado:

$$
R V=\left[1-\sum_{i=1}^{n} \sqrt{\frac{\left(d_{i}-D_{i}\right)^{2}}{d_{i}^{2}}}\right] \times 100
$$

siendo $n$ el número de muestras consideradas en cada registro, $d$ el sismograma observado y $D$ el sismograma sintético. El valor de $R V$ total es el promedio de los $R V$ de todas las componentes y estaciones que intervienen en el cálculo.

Tabla. 1. Acimut $\left(\right.$ Ac. $\left.^{\circ}\right)$, buzamiento $\left(\right.$ Buz. $\left.^{\circ}\right)$ y deslizamiento $\left(\right.$ Des. $\left.^{\circ}\right)$ de los planos nodales, momento sísmico escalar $\left(\mathbf{M}_{\mathbf{0}}\right)$, magnitud momento (Mw), porcentajes de fuente de doble par (\% D. Par), porcentaje de fuente dipolo compensado (\% Dipolo) y reducción de la varianza (\% RV) para el terremoto principal, premonitorio y réplica.

\begin{tabular}{|c|c|c|c|c|c|c|c|c|}
\hline Fecha Hora & Ac. $^{\circ}$ & Buz. $^{\circ}$ & Des. $^{\circ}$ & $\begin{array}{c}\mathbf{M}_{\mathbf{0}} \\
{[\mathbf{N ~ m}]}\end{array}$ & Mw & $\begin{array}{c}\text { \% } \\
\text { D. Par }\end{array}$ & $\begin{array}{c}\text { \% } \\
\text { Dipolo }\end{array}$ & $\begin{array}{c}\text { \% } \\
\text { RV }\end{array}$ \\
\hline 11-05-2011 15:05 & $127 ; 239$ & $72 ; 40$ & $126 ; 28$ & $6.10 \times 10^{15}$ & 4.5 & 72 & 28 & 77.65 \\
\hline $11-05-201116: 47$ & $230 ; 122$ & $64 ; 58$ & $37 ; 149$ & $4.63 \times 10^{16}$ & 5.1 & 97 & 3 & 74.47 \\
\hline $11-05-201120: 37$ & $215 ; 110$ & $71 ; 53$ & $39 ; 156$ & $7.33 \times 10^{14}$ & 3.9 & 81 & 19 & 81.16 \\
\hline
\end{tabular}

En la Figura 5 se muestra la representación de los tres mecanismos, donde el mecanismo principal se corresponde con una falla de desgarre con una ligera componente de falla inversa para los tres terremotos. En el terremoto principal, aparece una alta componente de mecanismo de doble par, que se reduce en el premonitorio y en la réplica, no siendo significativa. El eje de presión en los tres mecanismos tiene dirección N-S y es casi horizontal, la tensión tiene una dirección E-O. El plano nodal orientado en dirección NE-SO y con buzamiento hacia el NO, tiene valores de acimut y buzamiento compatibles con los de la falla de Alhama de Murcia (Figura 1), además, la serie de réplicas se alinea con su traza, por lo que se evidencia la asociación de estos sismos a dicha falla.

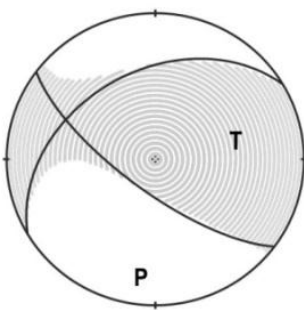

11 mayo 2011

15:05 (TU)

$4.5 \mathrm{Mw}$

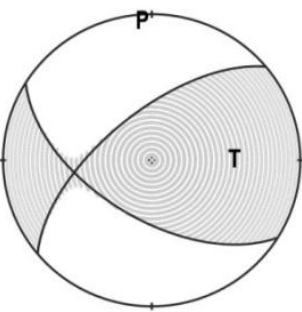

11 mayo 2011

16:47 (TU)

$5.1 \mathrm{Mw}$

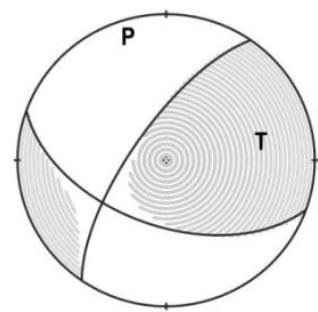

11 mayo 2011

20:37 (TU)

$3.9 \mathrm{Mw}$ 
Fig. 5. Mecanismos focales del terremoto principal, premonitorio y réplica obtenidos de la descomposición del tensor momento sísmico.

La localización en profundidad del centroide, se ha hecho mediante el análisis de la reducción de la varianza en la determinación del TMS a diferentes profundidades. El mejor resultado de reducción de la varianza se ha obtenido en las determinaciones a $4 \mathrm{~km}$ de profundidad para los tres TMS correspondientes a los sismos calculados.

En las Figuras 6, 7 y 8 se muestra la comparación entre los sismogramas observados en unidades de desplazamiento y los sintéticos calculados con los correspondientes TMS, para los terremotos de la Tabla 1: premonitorio, principal y réplica, respectivamente. Se observa que el ajuste es muy elevado, aunque el número de estaciones usadas depende de la magnitud del evento. Al disminuir la magnitud, la señal de largo periodo $(0.02-0.05 \mathrm{~Hz})$ se empieza a confundir con el ruido, lo que hace que algunas de las estaciones más alejadas no puedan utilizarse.

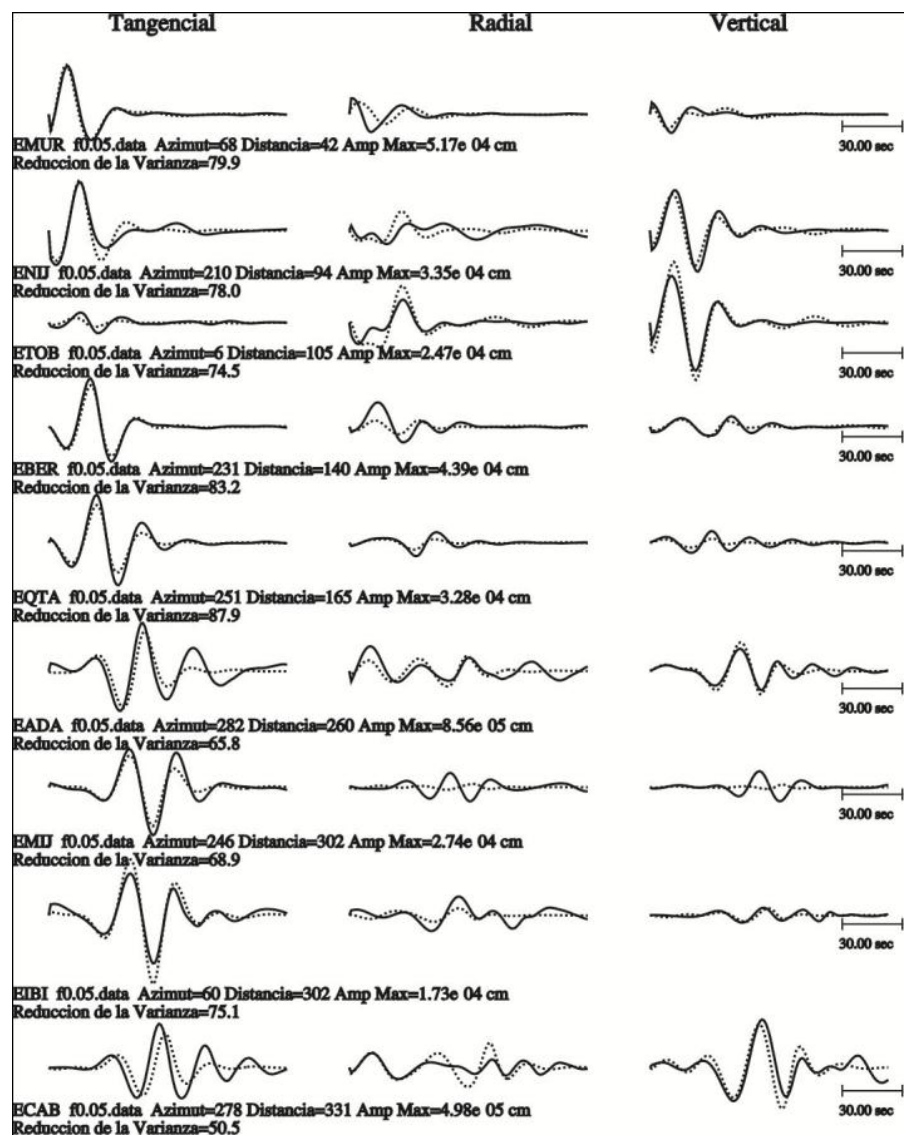


Fig. 6. Terremoto premonitorio del 11 de mayo de 2011, 15:05 T.U., 4,5 Mw. Ajuste entre sismogramas $(0.02-0.05 \mathrm{~Hz})$ observados y sintéticos (trazos), en desplazamiento, calculados con el tensor momento sísmico obtenido en el proceso de inversión.

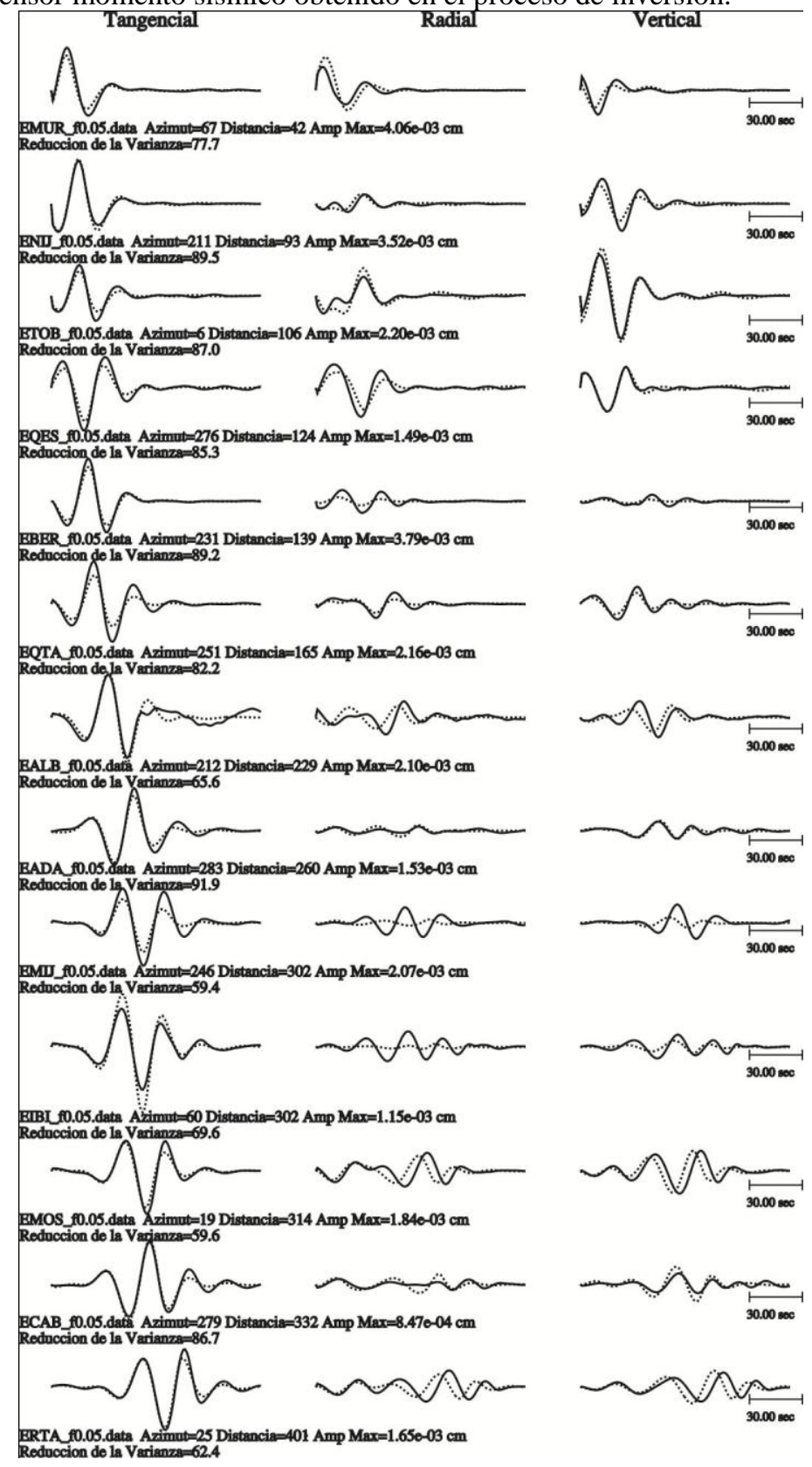


Fig. 7. Terremoto del 11 de mayo de 2011, 16:47 T.U., 5,1 Mw. Ajuste entre sismogramas (0.02-0.05 Hz) observados y sintéticos (trazos), en desplazamiento, calculados con el tensor momento sísmico obtenido en el proceso de inversión.

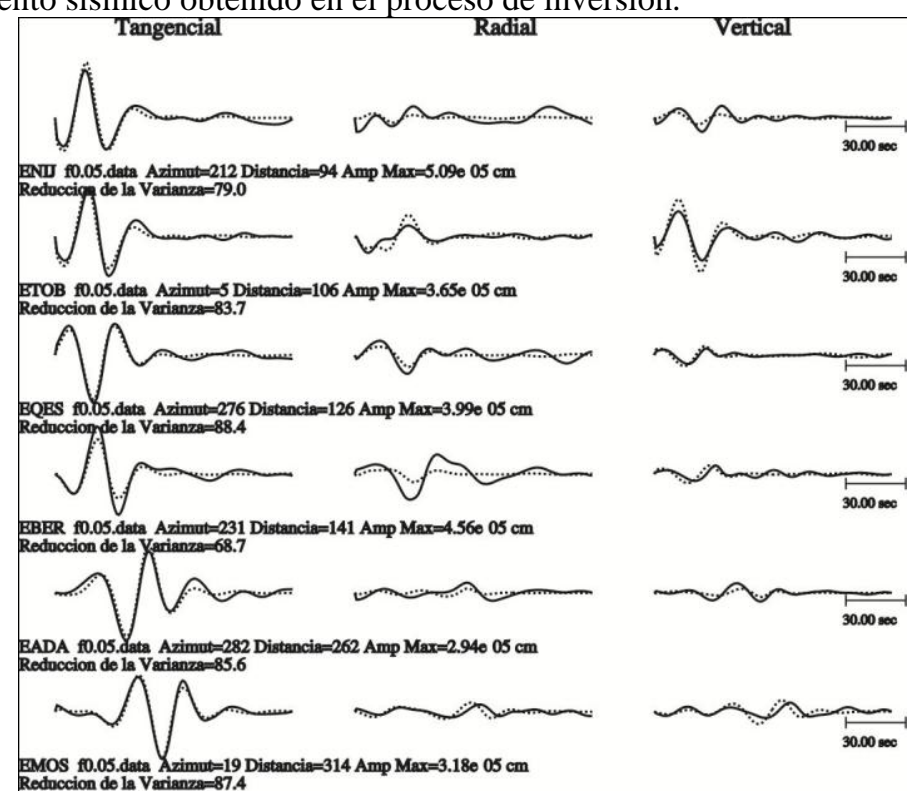

Fig. 8. Réplica del 11 de mayo de 2011, 20:37 T.U., 3,9 Mw. Ajuste entre sismogramas (0.02-0.05 Hz) observados y sintéticos (trazos), en desplazamiento, calculados con el tensor momento sísmico obtenido en el proceso de inversión.

\subsection{Inversión en una falla finita. Distribución del deslizamiento}

El proceso de inversión en una falla finita a distancia regional de Dreger y Kaverina (2000), basado en el desarrollo de Hartzell y Heaton (1983) para distancias telesísmicas, se inicia a partir del TMS que nos proporciona la magnitud momento y la orientación de los planos nodales, de los cuales uno será el plano de falla. La discriminación entre el plano de falla y el plano auxiliar, se hace repitiendo el proceso de inversión para ambos planos. El plano que produce un mejor ajuste entre observaciones y sintéticos, expresado con un mayor valor de la reducción de la varianza $R V$, corresponderá al plano de falla.

Con el fin de escalar el problema antes de discretizar el plano de falla, el valor de la magnitud momento nos permitirá, mediante las relaciones de Wells y Coppersmith (1994), obtener una aproximación a las dimensiones de la fractura. La fractura se discretiza dividiéndose en pequeñas subfracturas para realizar la integración a lo largo de toda la superficie. La integración se transforma en una integración en el tiempo, al considerar un modelo de evolución de la ruptura en el 
que en una subfractura se produce el deslizamiento cuando llega a ella el frente de onda.

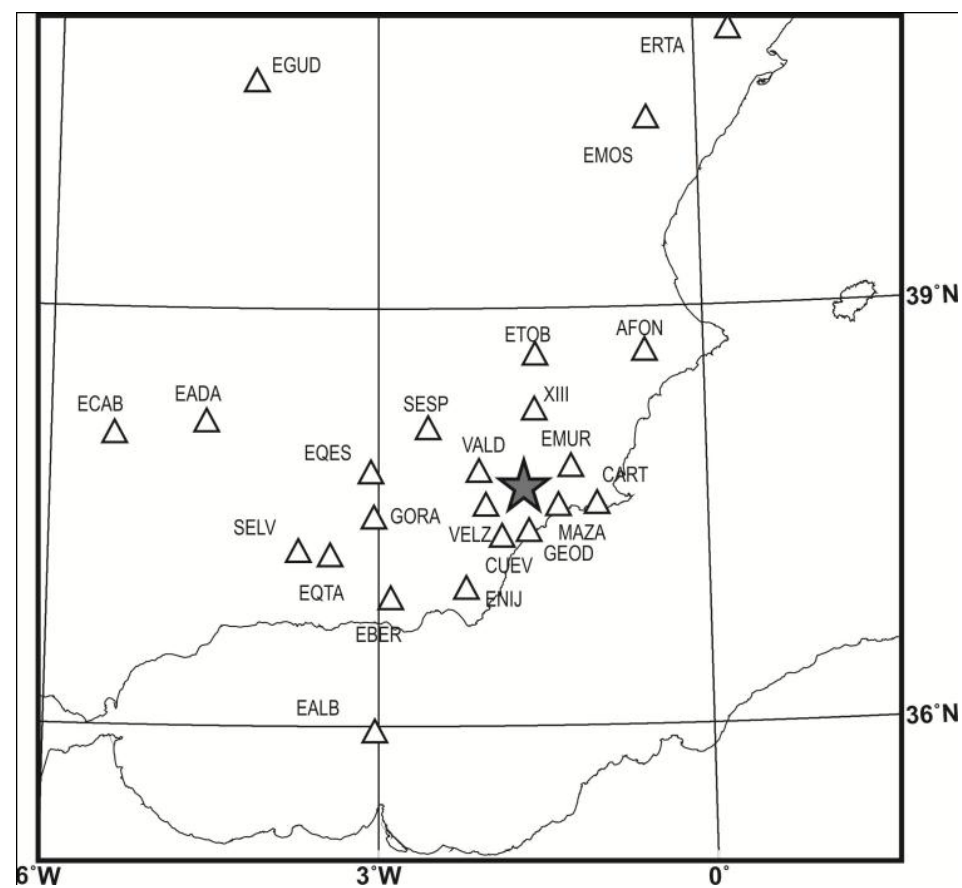

Fig. 9. Estaciones de banda ancha utilizadas en el cálculo de la distribución del deslizamiento a lo largo del plano de falla (Instituto Geográfico Nacional, Instituto Andaluz de Geofísica, Proyecto INDALO, Universidad de Alicante y consorcio ROA/UCM/Geofon) y localización del terremoto del 11 de mayo de 2011.

En el proceso de inversión es necesario disponer igualmente de las funciones de Green que representan la propagación por el medio y que ya habíamos utilizado en el cálculo del TMS.

Partiendo de los resultados obtenidos en el cálculo de TMS (Tabla 1), se ha calculado la distribución del deslizamiento en los dos posibles planos de falla para el terremoto principal. Consideramos una superficie de falla en la cual vamos a calcular el deslizamiento de $15 \times 15 \mathrm{~km}$, centrada en el hipocentro calculado y una discretización con tamaño de celda para las subfallas de $0,6 \times 0,6 \mathrm{~km}$. La superficie de cálculo considerada es bastante mayor que la que se corresponde para un terremoto de magnitud momento 5,1 $(3,7 \times 3,8 \mathrm{~km})$ según las relaciones de Wells y Coppersmith (1994), ya que hay que prever que como resultado de la inversión el deslizamiento no estuviese centrado en el hipocentro calculado.

Se han utilizado los datos de las estaciones de banda ancha de las redes permanentes del Instituto Geográfico Nacional, del Instituto Andaluz de Geofísica, 
incluidas para este último las estaciones temporales de su proyecto INDALO, Universidad de Alicante y consorcio ROA/UCM/Geofon, Figura 9, filtradas con un filtro pasobanda $0.02-0.10 \mathrm{~Hz}$.

Para resolver la dualidad sobre el plano de falla y para obtener la velocidad de ruptura, se calcula la distribución del deslizamiento para cada uno de los planos nodales y distintos valores de velocidad, desde 0,5 a $3,5 \mathrm{~km} / \mathrm{s}$, 95bteniendose para cada cálculo el valor de la reducción de la varianza. El resultado se muestra en la Figura 10, donde se aprecia de forma clara un mejor ajuste para el plano del mecanismo focal que buza hacia el noroeste $\left(\right.$ Ac. $=230^{\circ}$, Buz. $=64^{\circ}$, Des. $=37^{\circ}$ ), interpretado por su correlación con la neotectónica como plano de falla. Del análisis de la forma de la distribución del deslizamiento para cada velocidad de ruptura podemos deducir que el mismo es solamente coherente con un terremoto de magnitud 5,1 Mw para los cálculos realizados en el intervalo de 2 a $3 \mathrm{~km} / \mathrm{s}$. En este intervalo existe un máximo no muy bien definido de valor 2,4 $\mathrm{km} / \mathrm{s}$ que ha sido seleccionado como velocidad de ruptura.

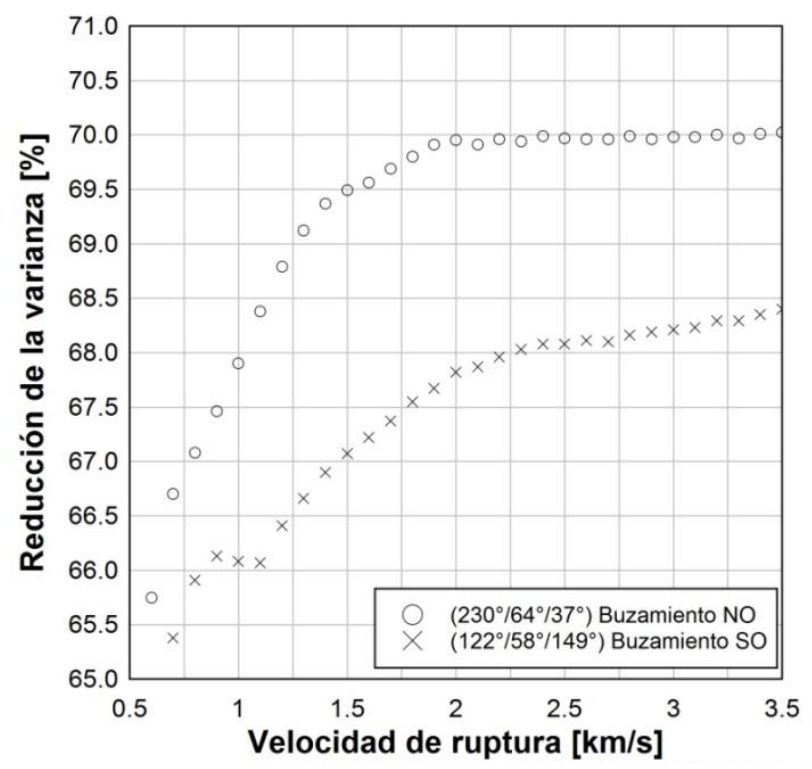

Fig. 10. Obtención del plano de falla y de la velocidad de ruptura mediante el análisis de la reducción de la varianza en función de la velocidad de ruptura para cada uno de los planos nodales. Este gráfico se obtiene mediante la repetición del proceso de inversión para cada uno de los escenarios.

Con el resultado de la velocidad de ruptura y confirmado el plano de falla, el resultado de la inversión nos proporciona la distribución del deslizamiento calculado para dicho plano de falla que se muestra en la Figura 11, presentando un 
deslizamiento máximo de $17,5 \mathrm{~cm}$ y un momento sísmico total de $6,21 \times 10^{17} \mathrm{~N} \mathrm{~m}$, correspondiente a una magnitud momento de 5,13 y una reducción de la varianza del $70 \%$. El deslizamiento en el inicio de la ruptura es de $4 \mathrm{~cm}$ y el deslizamiento medio de $4,7 \mathrm{~cm}$ se ajusta al calculado mediante la relación empírica de Somerville et al. (1999), que para una magnitud momento de 5,1 es de 5,6 cm.

Se aprecia en la Figura 11 que la ruptura que se inicia en el hipocentro, se dirige hacia el suroeste, hacia la ciudad de Lorca, de forma unilateral, mostrando ya un claro efecto de directividad.

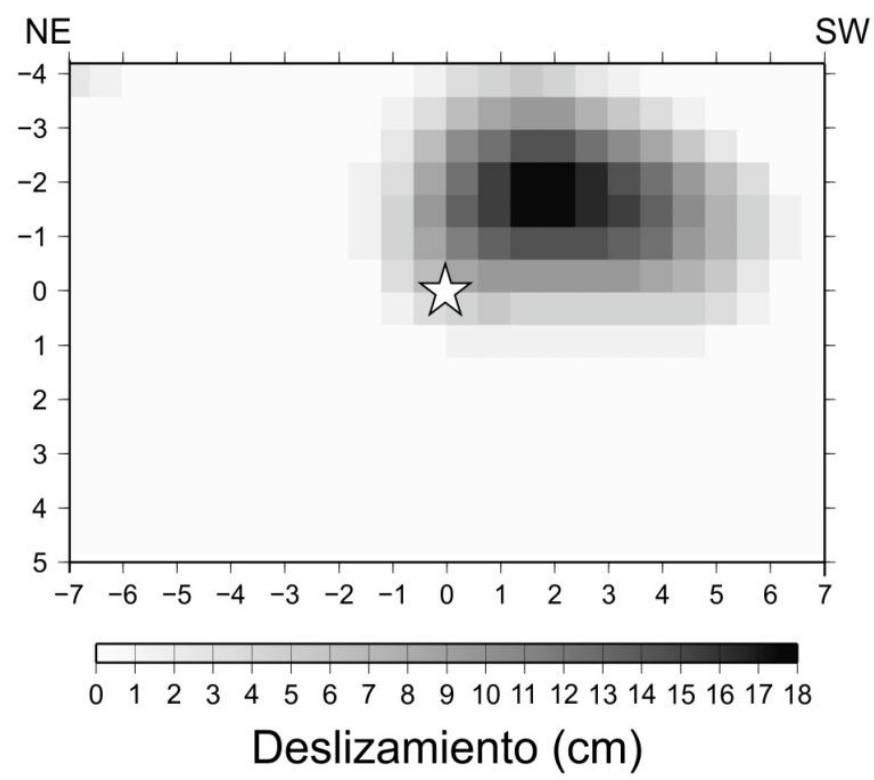

Fig. 11. Distribución del deslizamiento en cm a lo largo del plano de falla en el terremoto del 11 de mayo de 2011, 16:47 T.U., 5,1 Mw.

González et al. (2011), mediante interferometría de satélite, han obtenido un deslizamiento máximo de $20 \mathrm{~cm}$. Martínez-Díaz et al. (2012) con la misma técnica obtienen un deslizamiento máximo cosísmico de $15 \mathrm{~cm}$ con una distribución del mismo similar a la que se obtiene en el presente trabajo. Frontera et al. (2011) analizan datos GPS e interferometría satélite, obteniendo resultados del mismo orden de magnitud. Santoyo (2012) calcula la distribución del deslizamiento a partir de una única estación, el acelerógrafo de Lorca, asumiendo una velocidad de ruptura de $2,6 \mathrm{~km} / \mathrm{s}$ y un tiempo de subida de la dislocación de $1,15 \mathrm{~s}$, resultando un deslizamiento máximo de $135 \mathrm{~cm}$, muy superior a todos los obtenidos. 
VALDE

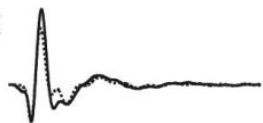

MAZAN
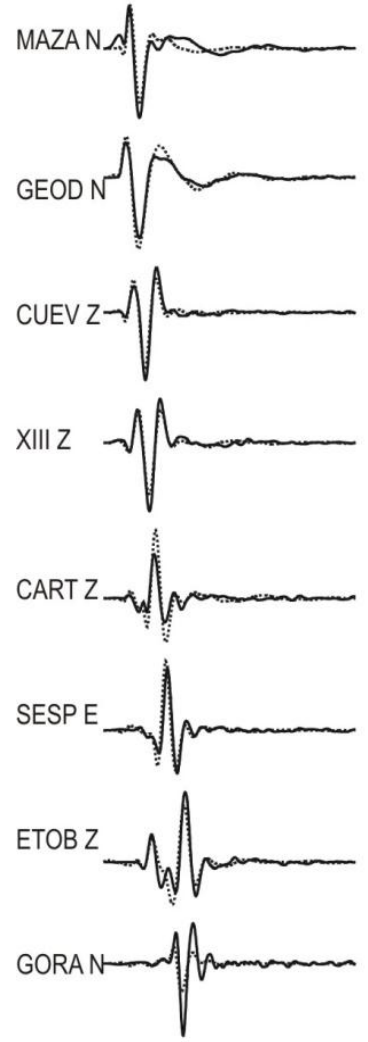

AFON E

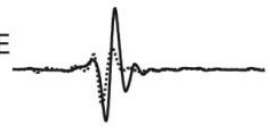

SELV N
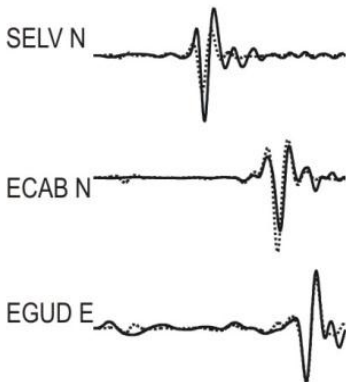

VELZ $N$

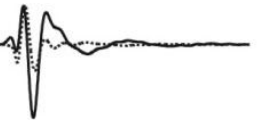

MAZAE
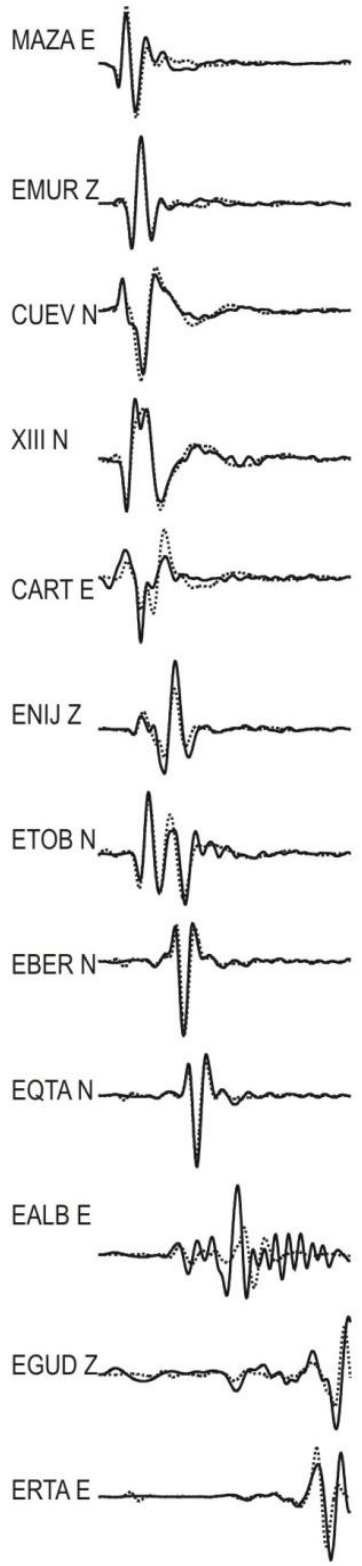
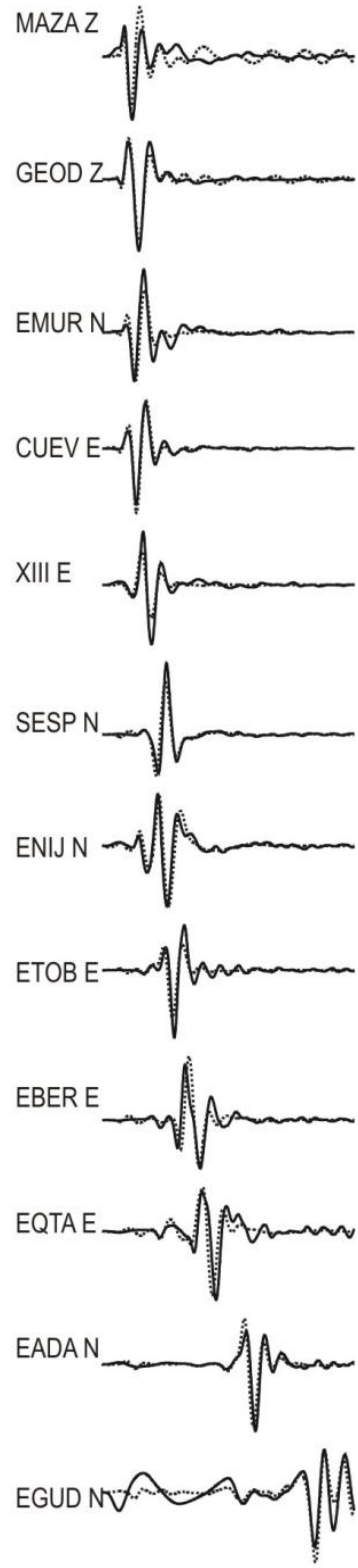

$100 \mathrm{~s}$

Fig. 12. Terremoto del 11 de mayo de 2011, 16:47 T.U., 5,1 Mw. Ajuste entre sismogramas $(0.02-0.10 \mathrm{~Hz})$ observados y sintéticos (trazos), en desplazamiento, calculados con la distribución del deslizamiento en el plano de falla, obtenido en el proceso de inversión. 
Con los resultados de la inversión, se han calculado sismogramas sintéticos y se comparan con los observados en la Figura 12 . La comparación se resuelve con una reducción de la varianza del $70 \%$, después de eliminar del proceso de cálculo algunas estaciones/componentes en las cuales la reducción de la varianza era menor del 50\%.

Nuestros resultados son obtenidos a partir de datos sísmicos únicamente y ya que estos datos son recibidos en tiempo real, nos permite que el proceso de inversión se pueda realizar a los pocos minutos de ocurrir el terremoto, con un tiempo de procesado de unos 20 minutos aproximadamente. Esto es posible ya que hemos generado previamente una biblioteca de funciones de Green de tal forma que no es necesario su cálculo en el momento de la inversión. En un procesado posterior, es conveniente combinar datos sísmicos y geodésicos con el fin de ajustar mejor los resultados con datos de deformaciones cosismicas directamente observadas en la superficie, Kaverina et al. (2002), Dreger et al. (2011).

La obtención de la distribución del deslizamiento a los pocos minutos de ocurrir un terremoto, permite simular de forma rápida, mediante procesos automáticos, el valor de la aceleración del movimiento del suelo y su velocidad, Dreger y Kaverina (2000), Dreger et al. (2005), Wu y Horiuchi (2008), obteniéndose así un mapa de sacudida sintético, que será de vital importancia para las primeras labores de emergencia.

\section{Análisis de la directividad}

Una vez resuelta de forma analítica la dualidad sobre el plano de falla, comprobado que el avance de la ruptura se realiza en la dirección de la falla de Alhama de Murcia, desde el hipocentro hacia Lorca, según refleja la distribución del deslizamiento y que el mismo se hace con una velocidad de ruptura de $2,4 \mathrm{~km} / \mathrm{s}$, vamos a realizar un análisis para obtener detalles de las características de esta directividad.

\subsection{Extracción del pulso de directividad}

Llamamos pulso a un registro de corta duración que se aprecia claramente en el sismograma de velocidad con una gran amplitud. Descartados los problemas instrumentales, una de las causas de estos pulsos es el efecto de la directividad en el campo próximo, Somerville et al. (1997). Cuando el mecanismo del terremoto es de falla de desgarre o falla inversa, el efecto de la directividad en el campo próximo se pone de manifiesto en la dirección normal a la falla, registrándose en esta componente y en estaciones muy próximas un pulso que se hace especialmente significativo en el registro de velocidad, Baker (2007), Baker y Asce (2008).

Varios autores han establecido relaciones entre el periodo del pulso de directividad y la magnitud del terremoto, por ejemplo: Mavroeidis y Papageorgiou (2003), Somerville (2003), Bray y Rodríguez-Marek (2004). 
Baker (2007) establece la relación:

$$
E\left[\ln T_{p}\right]=-5,78+1,02 \mathbf{M}
$$

donde $T_{p}$ es el periodo del pulso extraído, y $\mathbf{M}$ la magnitud momento. $E\left[\ln T_{p}\right]$ representa el valor medio de $\ln T_{p}$ para las dos componentes: en la dirección de la traza de la falla y en la dirección perpendicular.
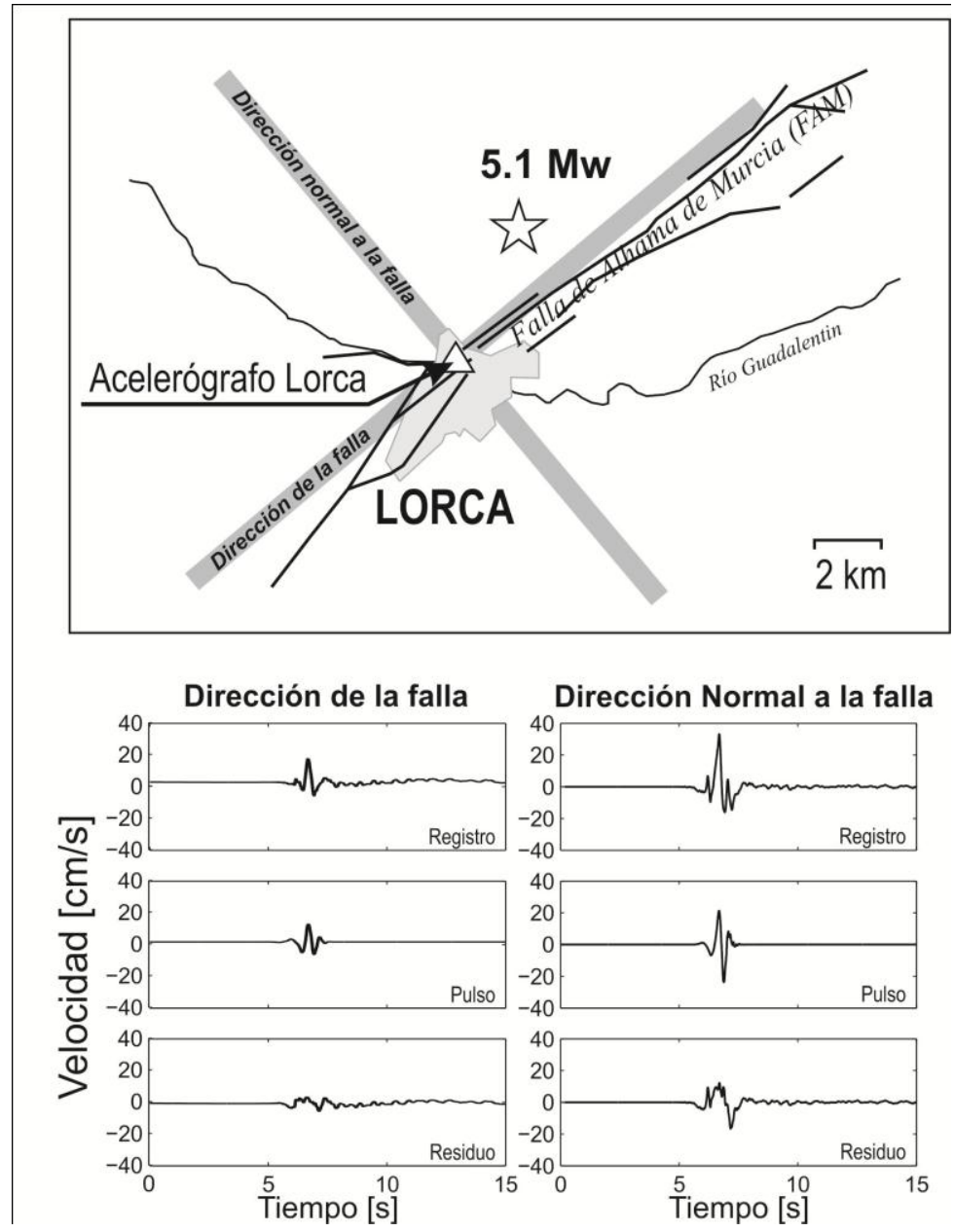

Fig. 13. Extracción del pulso de directividad con el método de Baker (2007) en el registro de velocidad del acelerógrafo de Lorca, en la dirección de la falla de Alhama de Murcia y en dirección perpendicular, para el terremoto del 11 de mayo de 2011, 16:47 T.U., 5,1 Mw. 
En nuestro caso, hemos utilizado el algoritmo de Baker (2007) para detectar y extraer el pulso de directividad del registro en velocidad, obteniendo su periodo, mediante el uso de la transformada wavelet. Este algoritmo clasifica el pulso extraído a partir de una biblioteca de pulsos clasificados previamente de forma manual, proporcionando un indicador de pulso en función del cociente entre la velocidad máxima del pulso y la velocidad máxima residual y el cociente entre la energía máxima del pulso y la energía máxima residual, después de extraído el pulso. La decisión final sobre si estamos ante un pulso de directividad o no, se toma cuando este indicador supera el valor de 0,85; si además al comparar las funciones acumuladas del cuadrado de la velocidad del registro original y la del pulso extraído se supera el $10 \%$ y como regla general, solamente se considerará pulso de directividad cuando el pico de velocidad sea mayor de $30 \mathrm{~cm} / \mathrm{s}$.

El resultado para el registro del terremoto principal de la serie en el acelerógrafo situado en Lorca, a $5 \mathrm{~km}$ del epicentro, se muestra en la Figura 13. En esta figura vemos el registro original del acelerógrafo de Lorca girado en la dirección de la falla de Alhama de Murcia y en la dirección normal, integrado para obtener velocidad, el pulso extraído con el algoritmo de Baker (2007) y el residuo que nos queda al restar del registro original el pulso detectado.

Para la componente normal a la dirección de la falla de Alhama de Murcia, el indicador de pulso es de 1 , con un periodo de $0,48 \mathrm{~s}$ y una velocidad máxima del pulso extraído de $33,2 \mathrm{~cm} / \mathrm{s}$. Para la componente en la dirección de la falla, el indicador es también 1, con un periodo de $0,67 \mathrm{~s}$ y velocidad máxima 14,6 cm/s. La magnitud momento deducida a partir del periodo del pulso es de 5,11; coincidente con la obtenida con el TMS y con la distribución del deslizamiento a lo largo del plano de falla.

Según el criterio de Baker (2007), en el registro del terremoto del 11 de mayo de 2011 en el acelerógrafo de Lorca estamos ante un pulso de directividad.

\subsection{Función temporal en la fuente}

La directividad de la ruptura puede estimarse también por la variación de la duración y amplitud de la función temporal en la fuente (FTF), calculada en una red de estaciones que cubran acimutalmente al epicentro. La FTF calculada en estaciones en la dirección de la falla responsable tiene una duración menor y una amplitud mayor que el cálculo en estaciones en la dirección contraria, debiéndose conservar el área. El acimut desde el epicentro a las estaciones con menor duración de la FTF define entonces la dirección de la ruptura.

Para determinar la FTF se ha utilizado el método de las funciones empíricas de Green, desarrollado por Hartzell (1978), que considera dos terremotos con el mismo hipocentro y mecanismo focal pero diferente magnitud. Varios autores han utilizado este método para estudiar como nosotros procesos de directividad en la ruptura, p.e.: Dreger (1994), Lay et al. (1994), Velasco et al. (1994), Cassidy (1995), Mori (1996), Dreger (1997), Ichinose et al. (1999), Abdel-Fattah (2002), Roumelioti et al (2003). 
El proceso de cálculo para cada una de las estaciones comienza con la integración de los sismogramas banda ancha de velocidad para obtener registro de desplazamiento. La diferencia de magnitudes entre los terremotos seleccionados, según Mori y Frankel (1990), debe estar entre 1,5 y 2 unidades de magnitud.

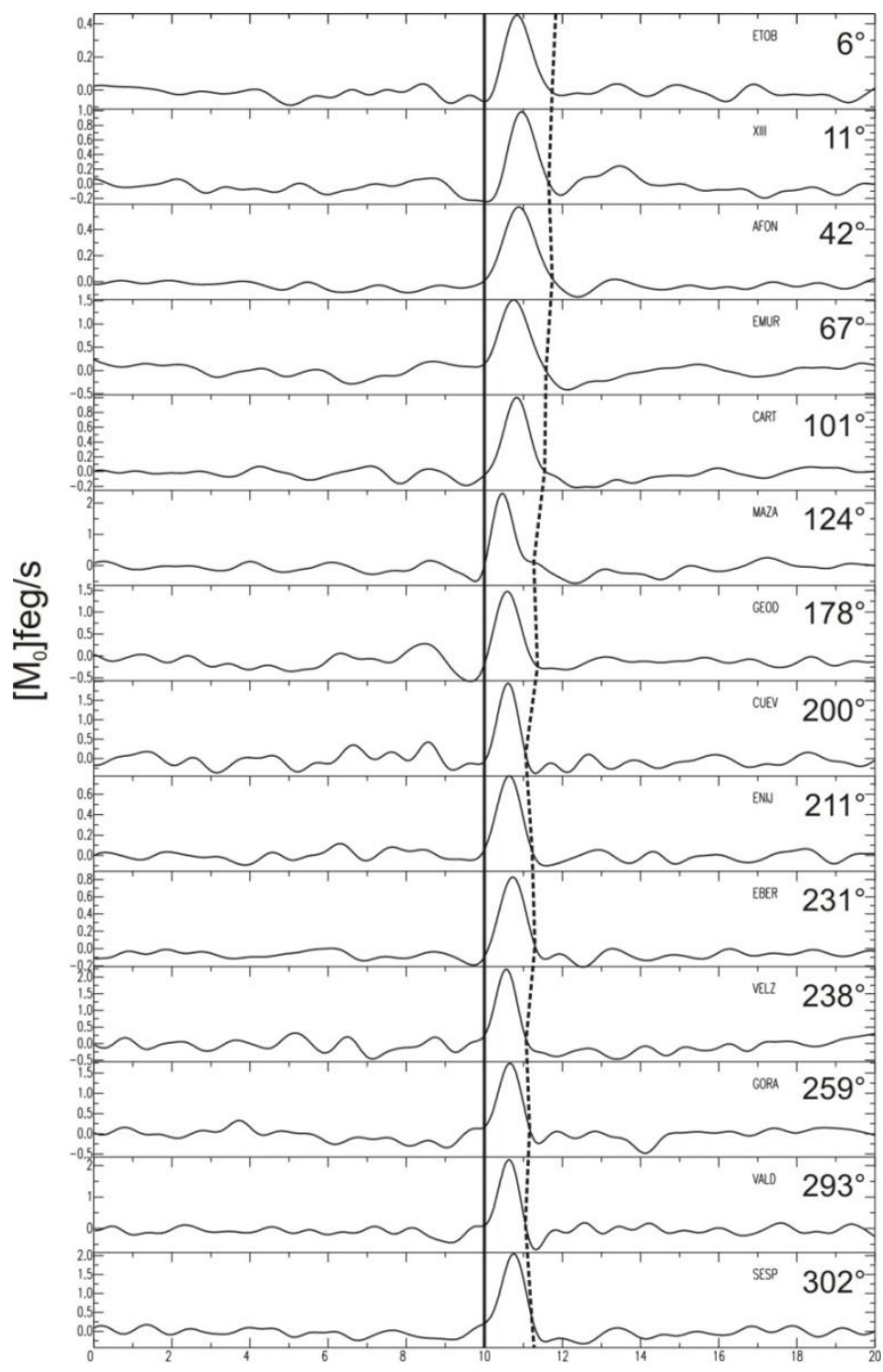

FTF [s]

Fig. 14. Función temporal en la fuente calculada para distintas estaciones mediante el método de la función empírica de Green, en el terremoto del 11 de mayo de 2011, 16:47 T.U., 5,1 Mw. 
En nuestro caso, hemos seleccionado como función empírica de Green la réplica ocurrida el día 11 de mayo a las 20:37 de magnitud 3,9 (Mw), con un mecanismo calculado prácticamente coincidente con el terremoto principal, Figura 5. A continuación hemos filtrado la señal con un filtro paso banda de frecuencia 0,05-1,0 Hz, Roumelioti et al. (2003). Con el fin de estabilizar la deconvolución, utilizamos un filtro de agua al 1\%, Clayton y Wiggins (1976).

El resultado obtenido se muestra en la Figura 14, donde se ha representado la FTF obtenida para una serie de estaciones que cubren acimutalmente el epicentro. La duración media de la FTF es de 1,5 s, pero se observa que esta se estrecha para direcciones próximas a la dirección y sentido del avance de la ruptura $\left(230^{\circ}\right)$ mientras que se ensancha en el sentido opuesto $\left(50^{\circ}\right)$, mostrando esto un claro efecto de directividad que se aprecia con más claridad en la representación acimutal de la Figura 15.

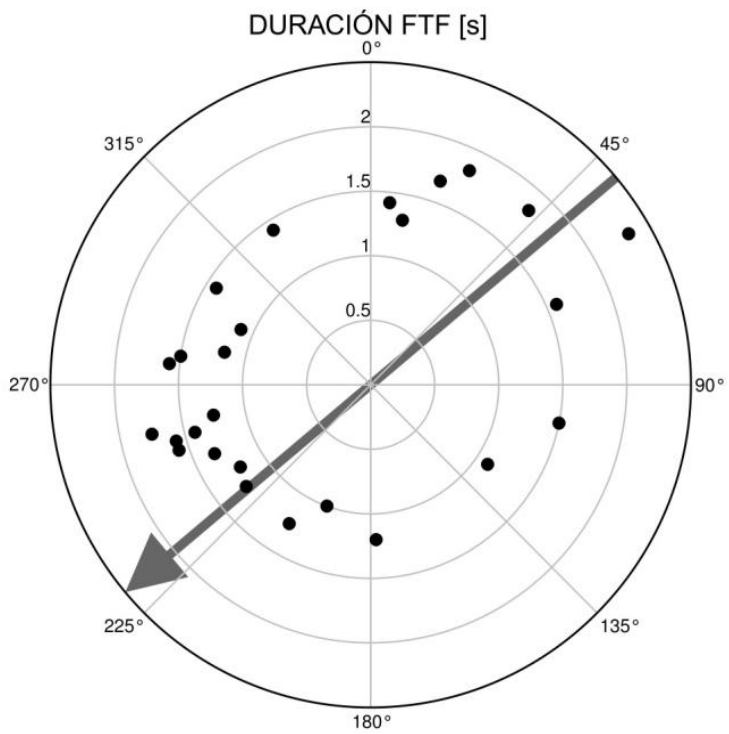

Fig. 15. Representación acimutal de la duración de la función temporal en la fuente para el terremoto del 11 de mayo de 2011, 16:47 T.U., 5,1 Mw, junto con la dirección del plano de falla obtenida en el cálculo del tensor momento sísmico.

López-Comino et al. (2012), a partir del análisis de las FTF calculadas con esta misma técnica, obtienen el mismo efecto de directividad, con un modelo de ruptura bilateral asimétrica que según ellos, ajusta mejor a las FTF que el simple modelo de ruptura unilateral. 


\subsection{Relación de amplitudes}

Para investigar la directividad de la fuente sísmica puede utilizarse también la comparación de amplitudes entre el terremoto principal, del que analizamos su directividad, y una o varias de las réplicas que no presenta este fenómeno, ya que al ser de baja magnitud la fuente pudiera considerarse puntual. Helmberger et al. (1992) utilizan este método para obtener la dirección de la ruptura y Jones el al. (1993) lo aplican para obtener las características del proceso de directividad de la ruptura del terremoto de Landers de magnitud 7,3 Mw. Recientemente, este método ha sido aplicado con éxito para estudiar la directividad en terremotos de magnitudes pequeñas e intermedias: Boatwright (2007), Seekins y Boatwright (2010), Tan y Helmberger (2010).

Hemos seleccionado 4 réplicas de magnitudes 3.9, 2.9, 2.8 y 2.7 para realizar el análisis. El registro de banda ancha en velocidad de cada terremoto, incluido el terremoto principal y cada estación/componente, lo transformamos para obtener la amplitud Wood-Anderson, haciendo primero la deconvolución del registro con su respuesta instrumental y luego la convolución con la respuesta equivalente de un equipo Wood-Anderson. A continuación integramos para obtener el registro en desplazamiento y rotamos las componentes NS y EO para obtener las componentes tangencial y radial.
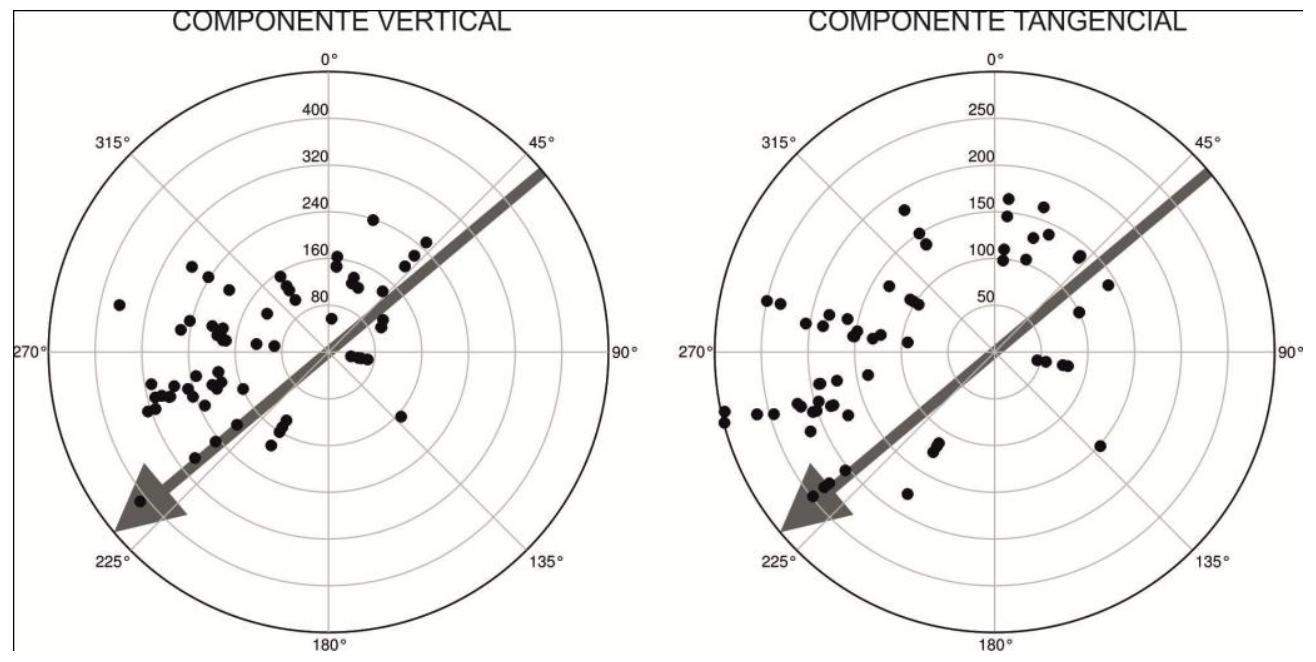

Fig. 16. Relación de amplitudes máximas en las componentes vertical (izquierda) y tangencial (derecha) del registro en desplazamiento Wood-Anderson para el terremoto principal y cuatro réplicas de magnitudes 3.9, 2.9, 2.8 y 2.7. Los cocientes se han normalizado a un valor de 100 para la estación EMUR. Se representa también la dirección del plano de falla obtenida en el cálculo del tensor momento sísmico. 
En la Figura 16 hemos representado el cociente de las amplitudes máximas medidas en el registro de desplazamiento Wood-Anderson para el terremoto principal y cada una de las réplicas en función del acimut de la estación, en las componentes vertical y tangencial, junto con la dirección del plano de falla determinado por el TMS. Con el fin de poder representar todos los terremotos en la misma figura, los cocientes se han normalizado a un cociente de valor 100 para la estación de EMUR. Tanto en la componente vertical como en la tangencial se puede ver claramente que la propagación de la ruptura se realiza en la dirección de la falla de Alhama de Murcia, en sentido SO, hacia la ciudad de Lorca.

Hemos realizado la misma operación con el terremoto principal y el premonitorio y los cocientes no indican la dirección de la ruptura. La explicación puede estar en que el sismo premonitorio de magnitud 4,5 Mw también pudiera presentar directividad, con lo que no se darían las condiciones necesarias de aplicación del método.

\section{Conclusiones}

Los grandes daños ocasionados por este terremoto de moderada magnitud fueron inicialmente relacionados con la influencia del efecto del suelo. Este efecto, que no puede descartarse como amplificador del movimiento del suelo, no es suficiente para alcanzar esos grados de daño, que por otra parte han tenido una localización no distribuida al azar sino siguiendo unas pautas muy precisas.

Se ha procedido a un análisis tanto de los datos de banda ancha pertenecientes a la Red Sísmica Nacional como los correspondientes a la Universidad de Alicante, consorcio ROA/UCM/Geofon, al Instituto Andaluz de Geofísica y al despliegue temporal de estaciones de banda ancha del proyecto INDALO. Asimismo se han utilizado los registros de la Red de Acelerógrafos en algunas partes de este trabajo.

Se ha observado una clara asimetría de los registros de aceleración que coincide con la distribución del daño observado a las mismas distancias que es mayor en la dirección SO del epicentro y mucho menor en la dirección NE.

La determinación del Tensor Momento Sísmico de los tres terremotos de mayor magnitud de la serie se ha realizado utilizando todos los datos de banda ancha disponibles. El resultado para los tres terremotos es una falla de desgarre con una ligera componente de falla inversa. El eje de presión en los tres mecanismos tiene dirección $\mathrm{N}-\mathrm{S}$ y es casi horizontal, la tensión tiene una dirección E-O. El plano nodal orientado en dirección NE-SO y con buzamiento hacia el NO, tiene valores de acimut y buzamiento compatibles con los de la falla de Alhama de Murcia, además, la serie de réplicas se alinea con su traza, por lo que se evidencia la asociación de estos sismos a dicha falla.

Siguiendo el proceso de inversión en una falla finita a distancia regional, descrito por Dreger y Kaverina (2000), se ha discretizado la falla en una malla de $0.6 \times 0.6 \mathrm{~km}$, probándose sobre ambos planos obtenidos en el cálculo del TMS. Variando la velocidad de ruptura se han realizado las comparaciones con los sismogramas reales expresándolas en tanto por ciento de la reducción de la va- 
rianza. El resultado obtenido es que el mejor ajuste lo proporciona el plano que buza al NO, con una velocidad de ruptura de $2,4 \mathrm{~km} / \mathrm{s}$. El resultado de la inversión en este plano presenta un deslizamiento máximo de $17,5 \mathrm{~cm}$ a $2 \mathrm{~km}$ de profundidad y un momento sísmico de $6,21 \times 10^{17} \mathrm{~N} \mathrm{~m}$. El deslizamiento medio es de $4,7 \mathrm{~cm}$ presentando en la superficie desplazamientos permanentes entre $0-4 \mathrm{~cm}$. El resultado de la inversión muestra una ruptura unilateral perfectamente compatible con la aparición del fenómeno de directividad. López-Comino et al. (2012), obtienen el mismo efecto de directividad, con un modelo de ruptura bilateral asimétrica. En nuestro caso, ninguno de los resultados obtenidos apunta hacia la ruptura bilateral.

A partir del registro de aceleración en la ciudad de Lorca se ha extraído el pulso de directividad mediante el método de Baker (2007) obteniendo su periodo mediante la utilización de la transformada de wavelet. El indicador para la componente normal a la dirección epicentro-acelerógrafo corresponde a un pulso de $0,48 \mathrm{~s}$ de periodo y una velocidad máxima de $33,2 \mathrm{~cm} / \mathrm{s}$, mientras que para la componente radial el periodo es de $0,67 \mathrm{~s}$ y la velocidad máxima de $14,6 \mathrm{~cm} / \mathrm{s}$. Estos valores están en consonancia con un pulso de directividad producido en la rotura sobre la falla de Alhama de Murcia.

Se ha estimado la función temporal en la fuente (FTF) a partir de considerar una réplica de magnitud 3,9 $\mathrm{Mw}$ e idéntico mecanismo focal al terremoto principal como función empírica de Green. El resultado es que la duración media de la FTF es de 1,5 s, observándose una disminución en la dirección coincidente con la dirección de ruptura $\left(230^{\circ}\right)$ mientras que sufre un aumento en la dirección opuesta $\left(50^{\circ}\right)$. Estos resultados son también indicadores inequívocos de que nos encontramos ante un fenómeno de directividad en el proceso de ruptura. Asimismo, se han comparado las amplitudes registradas en las distintas estaciones de la Red Sísmica Nacional del terremoto principal que presenta directividad con las correspondientes a algunas replicas que no muestran ese fenómeno. El resultado es que el cociente de amplitudes máximas del registro equivalente de WoodAnderson del terremoto principal y de esta replicas muestran que el máximo se corresponde con la dirección de la ruptura y el mínimo en la dirección opuesta. Es decir, tenemos otra verificación de que el terremoto de Lorca de 11 de Mayo de 2011 presento un fenómeno de directividad asociado a la ruptura y que su pulso es en gran medida, junto con los posibles efectos de amplificación por el tipo de suelo, el fenómeno responsable de las grandes aceleraciones observadas, así como de los daños ocasionados en Lorca.

Por último es necesario resaltar que el fenómeno de directividad observado en terremotos de mediana magnitud no puede considerarse extraño sino bastante habitual en ciertas fallas de la Península Ibérica, como puede ser la falla de Alhama de Murcia y que como ha ocurrido en Lorca puede afectar de forma grave a poblaciones muy próximas al epicentro. Por tanto, es imprescindible incluir en los estudios de peligrosidad sísmica este tipo de fuentes, que modificarán de forma importante los resultados obtenidos si no se considerase este fenómeno. 


\section{Agradecimientos}

Muchas de las figuras de este trabajo se han realizado con el software GMT de Wessel y Smith (1995). El tratamiento de los datos sísmicos digitales se ha realizado principalmente con SAC2000, Goldstein et al. (2003). Los autores agradecen a José Morales del Instituto Andaluz de Geofísica la facilidad para el uso de los datos de las estaciones sísmicas de banda ancha del proyecto INDALO, y por la misma razón a la Universidad de Alicante y al consorcio ROA/UCM/Geofon.

La metodología y parte de los resultados han sido discutidos de forma constructiva con Roula Roumelioti de Aristotle University of Thessaloniki y con Douglas Dreger de Berkeley Seismological Laboratory, que además nos ha proporcionado los programas de inversión para obtener el Tensor Momento Sísmico y la distribución del deslizamiento en el plano de falla. Agradecemos a Chandan Saikia por facilitarnos el software FKPROG para calcular las funciones de Green y a J.W. Baker de Stanford University por el programa para la extracción del pulso de directividad.

\section{Referencias bibliográficas}

ABDEL-FATTAH, A.K. (2002). Source characteristics of a moderate earthquake (M 4.9) using empirical Green's function technique. Ann. Geophys. 45, 575586.

BAKER, J.W. (2007). Quantitative classification of near-fault ground motions using wavelet analysis. Bull. Seismol. Soc. Amer. 97, 1486-1501, doi: $10.1785 / 0120060255$.

BAKER, J.W. \& ASCE, M. (2008). Identification of near-fault velocity pulses and prediction of resulting response spectra. Proceedings Geotechnical Earthquake Engineering and Structural Dynamics IV, Sacramento, CA, 10pp.

BENIOFF, H. (1955). Mechanism and strain characteritics of the White Wolf fault as indicated by the aftershock sequence. Calif. Div. Mines Bull. 171, 199202.

BENITO, B., CAPOTE, R., MURPHY, P., GASPAR-ESCRIBANO, J.M., MARTÍNEZ-DÍAZ, J.J., TSIGE, M., STICH, D., GARCÍA-MAYORDOMO, J., GARCÍA RODRÍGUEZ, M.J., JIMÉNEZ, M.E., INSUA-ARÉVALO, J.M., ÁLVAREZ-GÓMEZ, J.A. \& CANORA, C. (2007). An overview of the damaging and low magnitude Mw 4.8 La Paca earthquake on 29 January 2005: context, seismotectonics, and seismic risk implications for souutheast Spain. Bull. Seismol. Soc. Amer. 97, 671-690, doi: 10.1785/0120050150.

BEN-MENAHEM, A. (1961). Radiation of seismic surface-waves from finite moving sources. Bull. Seismol. Soc. Amer. 51, 401-435.

BEN-MENAHEM, A. \& SINGH, S.J. (1972). Computation of models of elastic dislocations in the earth. En Methods in Computational Physics, vol 12, editor B. Bolt. Academic Press.

BOATWRIGHT, J. (2007). The persistence of directivity in small earthquakes. Bull. Seismol. Soc. Amer. 97, 1850-1861, doi: 10.1785/0120050228. 
BOUSQUET, J.C. (1979). Quaternary strike-slip faults in southeastern Spain. Tectonophysics 52: 277-286.

BRAY, J.D. \& RODRÍGUEZ-MAREK, A. (2004). Characterization of forwarddirectivity ground motions in the near-fault región. Soil Dyn. Earthq. Eng. 24, 815-828.

BUFORN, E. \& SANZ DE GALDEANO, C. (2001). Focal mechanism of Mula (Murcia, Spain) earthquake of February 2, 1999. J. Seismol. 5, 277-280.

BUFORN, E., BENITO, B., SANZ DE GALDEANO, C., DEL FRESNO, C., MUÑOZ, D. \& RODRÍGUEZ, I. (2005). Study of the damaging earthquakes of 1911, 1999 and 2002 in the Murcia, southeastern Spain, region: seismotectonic and seismic-risk implications. Bull. Seismol. Soc. Amer. 95, 549-567, doi: 10.1785/0120040041.

BUFORN, E., CESCA, S., GODED, T., DEL FRESNO, C. \& MUÑOZ, D. (2006). The Bullas (Murcia, SE Spain) earthquake, 29 January 2005. J. Seismol. 10, 65-72, doi: 10.1007/s10950-006-2536-9.

CASSIDY, J.F. (1995). Rupture directivity and slip distribution for the Ms 6.8 earthquake of 6 April 1992, offshore British Columbia: an application of the empirical Green's function method using surface waves. Bull. Seismol. Soc. Amer. 85, 736-746.

ClAYTON, R.W. \& WIGGINS, R.A. (1976). Source shape estimation and deconvolution of teleseismic body waves. Geophys. J. R. Astr. Soc. 47, 151177.

DREGER, D. \& HELMBERGER, V. (1991). Complex faulting deduced from broadband modeling of the 28 February 1990 Upland earthquake $(\mathrm{ML}=5.2)$. Bull. Seismol. Soc. Amer. 81, 1129-1144.

DREGER, D. \& HELMBERGER, V. (1993). Determination of source parameters at regional distances with three-component sparse network data. J. Geophys. Res. 98, 8107-8125.

DREGER, D. (1994). Empirical Green's function study of the January 17, 1994 Northridge, California earthquake. Geophys. Res. Lett. 21, 2633-2636.

DREGER, D. (1997). The large aftershocks of the Northridge earthquake and their relationship to mainshock slip and fault-zone complexity. Bull. Seismol. Soc. Amer. 87, 1259-1266.

DREGER, D., UHRHAMMER, R., PASYANOS, M., FRANCK, J., \& ROMANOWICZ, B. (1998). Regional and far-regional earthquake locations and source parameters using sparse broadband networks: a test on the Ridgecrest sequence. Bull. Seismol. Soc. Amer. 88, 1353-1362.

DREGER, D. \& KAVERINA, A. (2000). Seismic remote sensing for the earthquake source process and near-source strong shaking: a case study of the October 16, 1999 Hector Mine earthquake. Geophys. Res. Lett. 27, 1941-1944.

DREGER, D., GEE, L. LOMBARD, P., MURRAY, M.H. \& ROMANOWICZ, B. (2005). Rapid finite-source analysis and near-fault strong ground motions: applications to the $2003 \mathrm{Mw}$ 6.5 San Simeon and 2004 Mw 6.0 Parkfield earthquakes. Seism. Res. Lett. 76, 40-48. 
DREGER, D., FORD, S.R. \& RYDER, I. (2011). Preliminary finite-source study of the February 21, 2008 Welss, Nevada earthquake. Nevada Bureau of Mines and Geology Special Publication 36, 147-156.

FAN, G. \& WALLACE, T. (1991). The determination of source parameters for small earthquakes from a single, very broadband seismic station. Geophys. Res. Lett. 18, 1385-1388.

FRONTERA, T., CONCHA, A., BLANCO, P., ECHEVERRIA, A., GOULA, X., ARBIOL, R., KHAZARADZE, G., PÉREZ, F. \& SURIÑACH, E. (2011). DInSAR coseismic deformation of the May $2011 \mathrm{Mw} 5.1$ Lorca earthquake, (Southern Spain). Solid Earth Discussions, 3, 963-974, doi: 10.5194/sed-3963-2011.

FUKULLAMA, E. \& DREGER, D. (2000). Performance test of an automated moment tensor determination system for the future "Tokai" earthquake. Earth Planets Space, 52, 383-392.

GOLDSTEIN, P., DODGE, D., FIRPO, M. \& MINNER, L. (2003). SAC2000: Signal processing and analysis tools for seismologist and engineers. Invited contribution to "The IASPEI International Handbook of Earthquake and Engineering Seismology", Edited by WHK Lee, H. Kanamori, P.C. Jennings, and C. Kisslinger, Academic Press, London.

GONZÁLEZ, P.J., TIAMPO, K.F., PALANO, M., CANNAVÒ, F. \& FERNÁNDEZ, J. (2011). Geodetically derived fault slip model for the May, 11th 2011 Lorca earthquake (SE, Spain). American Geophysical Union, Fall Meeting 2011, abstract \#G23A-0842.

HARTZELL, S.H. (1978). Earthquake aftershocks as Green's functions. Geopys. Res. Lett. 5, 1-4.

HARTZELL, S.H. \& HEATON, T.H. (1983). Inversion of strong ground motion and teleseismic waveform data for the fault rupture history of the 1979 Imperial Valley, California, earthquake. Bull. Seismol. Soc. Amer. 73, 1553-1583.

HELMBERGER, D.V., STEAD, R., HO-LIU, P. y DREGER, D. (1992). Broadband modeling of regional seismograms, Imperial Valley to Pasadena. Geophys. J. Int. 110, 42-54.

ICHINOSE, G., ANDERSON, J., SMITH, K., DE POLO, D., ANOOSHEHPOOR, R., SCHWEICKERT, R. \& LAHREN, M. (1999). The seismotectonics of the 30 October 1998 Incline Village, Nevada earthquake and its effects. Seism. Res. Lett. 70, 297-305.

IGME (2011). Instituto Geológico y Minero de España, Informe geológico preliminar del terremoto de Lorca del 11 de mayo del año 2011, 5.1 Mw. <http://www.igme.es> [consulta enero 2012].

IGN (2011). Instituto Geográfico Nacional, Informe del sismo de Lorca del 11 de mayo de 2011, revisado. <http://www.ign.es> [consulta junio 2012].

JONES, L.E., HOUGH, S.E. \& HELMBERGER, D.V. (1993). Rupture process of the June 28, 1992 Big Bear earthquake. Geophys. Res. Lett. 20, 1907-1910.

KAVERINA, A., DREGER, D. \& PRICE, E. (2002). The combined inversión of seismic and geodetic data for the source process of the 16 October $1999 \mathrm{Mw}$ 
7.1 hector mine, California, earthquake. Bull. Seismol. Soc. Amer. 92, 12661280.

LAY, T., RITSEMA, J., AMMON, C.J. \& WALLACE, T. (1994). Rapid sourcemechanism analysis of the April 29, 1993 Cataract Creek (Mw=5.3), northern Arizona earthquake. Bull. Seismol. Soc. Amer. 84, 451-457.

LÓPEZ-COMINO, J.Á., MANCILLA, F., MORALES, J. \& STICH, D. (2012). Rupture directivity of the 2011, Mw 5.2 Lorca earthquake (Spain). Geophys. Res. Lett. 39, Lo3301, doi: 10.1029/2011GL050498.

LUO, Y., TAN, Y., WEI, S., HELMBERGER, D., ZHAN, Z., NI, S., HAUKSSON, E. y CHEN, Y. (2010). Source mechanism and ruptura directivity of the 18 May $2009 \mathrm{Mw} 4.6$ Inglewood, California, earthquake. Bull. Seismol. Soc. Amer. 100, 3269-3277, doi: 10.1785/0120100087.

MANCILLA, F., AMMON, C.J., HERRMANN, R.B. \& MORALES, J. (2002). Faulting parameters of the 1999 Mula earthquake, southeastern Spain. Tectonophysics 354, 139-155.

MARTIN, G.R. Editor (1994). Proc. Of the NCEER/SEAOC/BSSC workshop on site response during earthquakes and seismic code provisions. University of $\mathrm{S}$. Calif., Los Angeles.

MARTÍNEZ-DÍAZ, J.J. (2002). Stress field variation related to fault interaction in a reverse oblique-slip fault: the Alhama de Murcia fault, Betic Cordillera, Spain. Tectonophysics 356, 291-305.

MARTÍNEZ-DÍAZ, J.J., LÓPEZ, C., CAPOTE, R., CARREÑO, E. \& MARTÍNASÍN, A. (2002). Mecanismo focal del terremoto de Lorca de 23 de agosto de 2000 (mb 3.9). Ejemplo de terremoto extensional bajo régimen compresivo. $3^{a}$ Asamblea Hispano-Portuguesa de Geodesia y Geofísica I: 407-411.

MARTÍNEZ-DÍAZ, J.J., BEJAR-PIZARRO, M., ÁLVAREZ-GÓMEZ, J.A., MANCILLA, F., STICH, D., HERRERA, G. \& MORALES, J. (2012). Tectonic and seismic implications of an intersegment ruptura. The damaging May 11th $2011 \mathrm{Mw} 5.2$ Lorca, Spain, earthquake. Tectonophysics doi: 10.1016/j.tecto.2012.04.010.

MAVROEIDIS, G.P. \& PAPAGEORGIOU, A.S. (2003). A mathematical representation of near-fault ground motions. Bull. Seismol. Soc. Amer. 93, 10991131.

MEZCUA, J. \& MARTÍNEZ SOLARES, J.M. (1983). Sismicidad del área IberoMogrebí. Pub. Instituto Geográfico Nacional, 203, 302 pp.

MEZCUA, J., HERRAIZ, M. \& BUFORN, E. (1984). Study of the 6 June 1977 Lorca (Spain) earthquake and its aftershocks sequence. Bull. Seismol. Soc. Amer. 74, 167-179.

MORI, J. \& FRANKEL, A. (1990). Source parameters for small events associated with the 1986 North Palm Springs, California, earthquake determined using empirical Green functions. Bull. Seismol. Soc. Amer. 80, 278-295.

MORI, J. (1996). Rupture directivity and slip distribution of the M 4.3 foreshock to the 1992 Joshua Tree earthquake, southern California. Bull. Seismol. Soc. Amer. 86, 805-810. 
NAVARRO, M., GARCÍA-JEREZ, J.A., ALCALÁ, F.J., VIDAL, F., ENOMOTO, T., LUZÓN, F. \& CREUS, C. (2008). Vs30 structure of Lorca town (SE Spain) from ambient noise array observations. Proceedings $31^{\text {st }}$ General Assembly of European Seismological Commission, Crete, Greece: 288-295.

NCSE (2002). Norma sismorresistente: parte general y edificación. Boletín Oficial del Estado 244, 35898-35967.

ROUMELIOTI, Z., DREGER, D., KIRATZI, A. \& THEODOULIDIS, N. (2003). Slip distribution of the 7 September 1999 Athens earthquake inferred from an empirical Green's function study. Bull. Seismol. Soc. Amer. 93, 775-782.

RUEDA, J. \& MEZCUA, J. (2005). Near-real-time seismic momento-tensor determination in Spain. Seism. Res. Lett. 76, 455-465.

RUEDA, J., MEZCUA, J. \& GARCÍA BLANCO, R.M. (2011). Directivity effects of the May 11, 2011 Lorca (Spain) Mw=5.1 earthquake. American Geophysical Union, Fall Meeting 2011, abstract \#S52B-2277.

SAIKIA, C.K. (1994). Modified frecuency-wavenumber algorithm for regional seismograms using Filon's quadrature: modeling of $\mathrm{Lg}$ waves in eastern North America. Geophys. J. Int. 118, 142-158.

SANTOYO, M.A. (2012). Finite fault analysis and near field dynamic strains and rotations due to the 11/05/2011 (Mw5.2) Lorca earthquake, south-eastern Spain. Bull. Earthq. Eng. (enviado).

SEEKINS, L.C. \& BOATWRIGHT, J. (2010). Rupture directivity of moderate earthquakes in northern California. Bull. Seismol. Soc. Amer. 100, 1107-1119, doi: $10.1785 / 0120090161$.

SHAHI, S.K. \& BAKER, J.W. (2010). Signal processing and probabilistic seismic hazard analysis tools for characterizing the impact of near-fault directivity. Joint conference proceedings: $7^{\text {th }}$ International Conference on Urban Engineering and $5^{\text {th }}$ International Confernece on Earthquake Engineering, Tokyo, Japan.

SOMERVILLE, P.G., SMITH, N.F., GRAVES, R.W., \& ABRAHAMSON, N.A. (1997). Modification of empirical strong ground motion attenuation relations to include the amplitude and duration effects of rupture directivity. Seismol. Res. Lett. 68, 199-222.

SOMERVILLE, P.G., IRIKURA, K., GRAVES, R., SAWADA, S., WALD, D., ABRAHAMSON, N., IWASAKI, Y., KAGAWA, T., SMITH, N., \& KOWADA, A. (1999). Characterizing crustal earthquake slip models for the prediction of strong ground motion. Seismol. Res. Lett. 70, 59-80.

SOMERVILLE, P.G. (2003). Magnitude scaling of the near fault rupture directivity pulse. Phys. Earth Planet. Inter. 137: 12.

STICH, D., AMMON, C.J., \& MORALES, J. (2003). Moment tensor solutions for small and moderate earthquakes in the Ibero-Maghreb región. J. Geophys. Res. 108, 2148, doi: 10.1029/2002JB002057. 
STICH, D., SERPELLONI, E., MANCILLA, F. \& MORALES, J. (2006). Kinematics of the Iberia-Maghreb plate contact from sseismic momento tensors and GPS observations. Tectonophysics 426: 295-317.

TAN, Y. \& HELMBERGER, D. (2010). Rupture directivity characteristics of the 2003 Big Bear sequence. Bull. Seismol. Soc. Amer. 100, 1089-1106, doi: $10.1785 / 0120090074$.

TOTHONG, P., CORNELL, C.A. \& BAKER, J.W. (2007). Explicit-directivitypulse inclusion in probabilistic seismic hazard analysis. Earthq. Spectra 23, 867-891.

VELASCO, A.A., AMMON, C.J. \& LAY, T. (1994). Empirical Green function deconvolution of broadband surface waves: rupture directivity of the 1992 Landers, California (Mw=7.3), earthquake. Bull. Seismol. Soc. Amer. 84, 735750.

VISSERS, R.L.M. \& MEIJNINGER, B.M.L. (2011). The 11 May 2011 earthquake at Lorca (SE Spain) viewed in a structural-tectonic context. Solid Earth, 2, 199-204, doi: 10.5194/se-2-199-2011.

WALTER, W.R. (1993). Source parameters of the June 29, 1992 Little Skull mountain earthquake from complete regional waveforms at a single station. Geophys. Res. Lett. 20, 403-406.

WELLS, D.L. \& COPPERSMITH, K.J. (1994). New empirical relationships among magnitude, rupture length, rupture width, rupture area, and surface displacement. Bull. Seismol. Soc. Amer. 84, 974-1002.

WESSEL, P., \& SMITH, W.H.F. (1995). New version of the Generic Mapping Tools released. Eos, Transactions of the American Geophysical Union 76: 329.

WU, C. \& HORIUCHI, S. (2008). Automatic determination of source parameters of the 2007 Noto Hanto earthquake. Earth Planets Space, 60, 1053-1057. 